\title{
Trajetórias periódicas em sistemas de controle afins
}

\section{Leandro Sebben Bellicanta}

\author{
TESE APRESENTADA \\ $\mathrm{AO}$ \\ INSTITUTO DE MATEMÁTICA E ESTATÍSTICA \\ DA \\ UNIVERSIDADE DE SÃO PAULO \\ PARA \\ OBTENÇÃO DO GRAU DE DOUTOR \\ EM \\ MATEMÁTICA APLICADA \\ Área de concentração: Matemática Aplicada. \\ Orientador: Prof. Dr. Pedro Aladar Tonelli.
}

Durante a realização deste trabalho o autor recebeu suporte financeiro da CAPES

Sāo Paulo, Agosto de 2002. 


\section{Trajetórias Periódicas em Sistemas de Controle Afins}

Este exemplar corresponde a versão final da tese de doutorado defendida por Leandro Sebben Bellicanta, devidamente corrigida e aprovada pela comissão julgadora.

São Paulo, Agosto de 2002.

Banca examinadora:

- Prof. Dr. Carlos José Braga Barros (UEM)

- Prof. Dr. Luciano Barbanti (IME-USP)

- Prof. Dr. Luiz Antonio Barrera San Martin (IMECC-UNICAMP)

- Prof. Dr. Paulo Sérgio Pereira da Silva (EP-USP)

- Prof. Dr. Pedro Aladar Tonelli (IME-USP) 
Aos meus pais, Anastácio e Zilá, pelo apoio e pelos valores que me ensinaram a cultivar.

$$
\text { À Alice, }
$$

pelo tanto que foi importante na minha vida. 
A mente humana é simultaneamente

a fonte e, se orientada de forma

apropriada, a solução de quase todos os nossos

problemas. Os que adquirem grande

erudição mas não têm bom coração correm

o risco de serem atormentados por

ansiedades e inquietações que resultam de desejos que não podem ser realizados.

Inversamente, a compreensão genuína dos valores espirituais tem o efeito oposto.

Dalai-Lama 


\section{Agradecimentos}

Gostaria de agradecer a todos que, de alguma forma, contribuiram positivamente para que este trabalho chegasse ao fim. Gostaria de registrar aqui meus agradecimentos aos colegas do Departamento de Matemática da Furg pelo apoio irrestrito que têm me prestado todos estes anos. Em particular, gostaria de agradecer ao colega Leivas e ao colega Mário Retamoso, pela leitura cuidadosa da versão preliminar desta tese, e ao colega Sebastião, pelas discussões sobre sistemas de controle que acabaram motivando meus estudos de doutorado.

Da mesma forma, gostaria de agradecer aos professores e funcionários do Instituto de Matemática e Estatística da Universidade de São Paulo, pois tiveram um papel importante nesta fase da minha carreira profissional.

Aos colegas Bárbara, Caputi, Cecília, Cibele, Claus, Cláudia, Domingos, Hernan, Jairo, Juan, Maité, Major, Paola, Raul, Sandra, Sônia e Washington, pelo convívio e pelos momentos de alegria e descontração, momentos estes que foram fundamentais para o equilíbrio do meu espírito e desta forma me ajudaram muito. Agradeço também ao Sandro, da cantina do IME, pela parceria nas corridas de final de tarde pelo campus da USP. Um agradecimento especial vai para o colega Mário Salles e para os casais Lurdinha e Osmar e Irene e Zé, que me acolheram no aconchego de seus lares durante vários dias. Para os colegas Fernando e Gláucio, agradeço pelas discussões matemáticas que muito me ajudaram a esclarecer certos pontos deste trabalho.

Aos professores Paulo Cordaro e Pedro Tonelli, pela confiança que depositaram em mim.

À Alice que esteve sempre ao meu lado e muito me ajudou a enfrentar a difícil tarefa de concluir este doutorado e a quem devo muitos dos momentos felizes que vivi em São Paulo. 


\section{Resumo}

Neste trabalho, consideram-se sistemas de controle afins, definidos em uma variedade diferenciável de dimensão finita $M$. O conjunto $\mathcal{P} \subset M$ dos pontos pelos quais passa alguma trajetória periódica do sistema de controle é particionado em classes de equivalência, onde os pontos equivalentes são os que pertencem a uma mesma trajetória periódica. Estas classes de equivalência são denominadas de Conjuntos de Controlabilidade Total. Mostra-se que o $\omega$-limite dos conjuntos de acessibilidade em tempo $t$ de pontos pertencentes ao mesmo conjunto de controlabilidade total não muda.

Da mesma forma, o conjunto $\mathcal{R} \subset M$ dos pontos recorrentes do sistema de controle é particionado em classes de equivalência, que são chamadas de Conjuntos de Controlabilidade Aproximada. Estes conjuntos são comparados com os "Control Sets", conceito introduzido por L. Arnold e W. Kliemann [2], e estudado com mais detalhes em [6], [7], [10]-[17], [38], [39], [44] dentre outros.

Conforme [10], associa-se um fluxo no espaço $\mathcal{U} \times M$ ao sistema de controle, onde $\mathcal{U}$ é o conjunto de controles admissíveis do sistema. Considera-se o levantamento dos conjuntos de controlabilidade total de $M$ para o conjunto $\mathcal{U} \times M$ e mostra-se que o fecho destes levantamentos são topologicamente mixing para o fluxo em $\mathcal{U} \times M$.

No capítulo 5, faz-se um estudo do conjunto $\mathcal{P}$ para o caso em que o sistema de controle é uma perturbação de um sistema de controle linear hiperbólico.

\section{Abstract}

In this work is considered smooth control-affine systems on a finite dimensional manifold $M$. The set $\mathcal{P} \subset M$ of periodic points for the control systems is partitioned in equivalence classes, where two points $x, y \in M$ are equivalents if $x$ and $y$ are in the same periodic trajectory. These equivalence classes had been called "Holding Sets" by E. Roxin [34]. We prove that the $\omega$-limit of the attainable set at time $t$ does not change for points in the same holding set.

In the same way, the set $\mathcal{R} \subset M$ of recurrent points for the control system is partitioned in another kind of equivalence classes. These equivalence classes are compared with the "control sets", concept introduced by L. Arnold and W. Kliemann [2] and studied in many others works, for example, [6], [7], [10]-[17], [39].

In accordance with [10], it is associated with the control system a dynamical system on $\mathcal{U} \times M$, where $\mathcal{U}$ is the set of admissible control functions. We lift the holding sets of $M$ to the set $\mathcal{U} \times M$ and prove that the closure of these lifts are topologically mixing for the dynamical system on $\mathcal{U} \times M$.

In the chapter 5 is studied the set $\mathcal{P}$ for perturbation of the hyperbolic linear control systems. 


\section{Conteúdo}

$\begin{array}{ll}\text { Introdução } & 7\end{array}$

1 Preliminares 12

1.1 O Sistema de Controle . . . . . . . . . . . . . . . . . . . . 12

1.2 Conjuntos de Acessibilidade e Controlabilidade . . . . . . . . . 14

1.3 Conjuntos de Controlabilidade Total . . . . . . . . . . . . . . 19

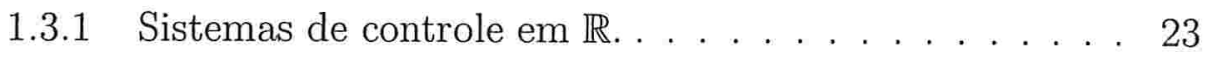

2 Comportamento Assintótico de $\mathcal{O}_{t}^{+}(x) . \quad 27$

2.1 Conjuntos de Controlabilidade Total e o Comportamento As-

sintótico dos Conjuntos de Acessibilidade. . . . . . . . . . 28

2.2 Recorrência . . . . . . . . . . . . . . . . . . . . 33

2.3 Conjuntos Controláveis. . . . . . . . . . . . . . . . . . . . 36

2.4 Notas . . . . . . . . . . . . . . . . . . . . . . 41

3 O Sistema Dinâmico em $\mathcal{U} \times M$. 42

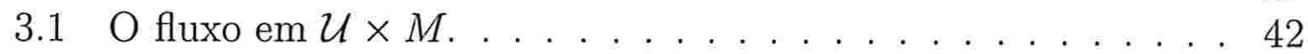

3.2 O levantamento de $\mathcal{P}$. . . . . . . . . . . . . . . . . . . . . 44

3.3 Notas. . . . . . . . . . . . . . . . . . . . . . . . . . . . . . . 49

4 Perturbação de sistemas lineares. $\quad 50$

4.1 Sistemas Lineares . . . . . . . . . . . . . . . . . . . . . . 50

4.2 Perturbação de sistemas lineares . . . . . . . . . . . . . . . . 55

4.3 Notas . . . . . . . . . . . . . . . . . . . . . . . . . 64 


\section{Introdução}

Este trabalho é um estudo da estrutura do conjunto das trajetórias periódicas de um sistema de controle e das relações existentes entre este conjunto e o comportamento assintótico do conjunto de acessibilidade do sistema. Como veremos, o conjunto de trajetórias periódicas está relacionado com a controlabilidade de certos subconjuntos do espaço de estados e também está relacionado a noções de recorrência em um sistema dinâmico topológico associado ao sistema de controle.

A presente tese tem como referência os trabalhos de Fritz Colonius e Wolfgang Kliemann, [10] e [11], que usaram o conceito de conjuntos controláveis e um sistema dinâmico topológico associado ao sistema de controle, o qual chamaram de sistema dinâmico de controle, para relacionar propriedades de recorrência do sistema dinâmico com propriedades de controlabilidade do sistema de controle. O ponto de vista do presente trabalho permite evitar, em alguns casos, a hipótese de acessibilidade local dos sistemas de controle, bem como a hipótese que os conjuntos controláveis tenham interior não vazio.

\section{Sistemas de Controle Afins.}

Suponha que em uma variedade diferenciável $M$, de dimensão finita, tenhamos um sistema dinâmico (com tempo contínuo) definido por uma equação diferencial

$$
\dot{x}=X_{0}(x) .
$$

A partir disso, considere a família de equações diferenciais

$$
\dot{x}=X_{0}(x)+\sum_{i=1}^{m} u_{i}(t) X_{i}(x)
$$


onde $X_{i}, i=1, \ldots, m$ são campos de vetores em $M$ e $u_{i}, i=1, \ldots, m$ são funções reais de variável real, que pertencem a um certo conjunto $\mathcal{U}$ de funções admissíveis. A princípio, o conjunto $\mathcal{U}$ das funções admissíveis depende do contexto considerado, podendo apresentar uma série de restrições. Por exemplo, se a família (1) fosse interpretada como uma perturbação não estacionária de uma equação diferencial ordinária pelos campos de vetores $X_{1}, \ldots, X_{m}$, o conjunto $\mathcal{U}$ representaria os possíveis ruídos e poderia ser considerado como um subconjunto de $L^{\infty}(\mathbb{R}, U)$ onde $U$ é um conjunto limitado do $\mathbb{R}^{m}$.

Outra forma de interpretar (1) é como um sistema de controle, onde os campos de vetores $X_{1}, \ldots, X_{m}$ modelam a parte do sistema que podemos controlar e o conjunto $\mathcal{U}$ denota os controles admissíveis para o sistema. Sistemas de controle deste tipo são chamados de sistemas de controle em malh a aberta. Neste caso, o conjunto de controles admissíveis poderia ser, por exemplo, um subconjunto das funções contínuas ou das funções constantes por partes. $\mathrm{Na}$ teoria moderna de sistemas de controle, os controles admissíveis usualmente são elementos do conjunto $\mathcal{U}=\left\{u: \mathbb{R} \rightarrow \mathbb{R}^{m}\right.$ mensurável : $u(t) \in U$ quase sempre $\}$ ou do conjunto $\mathcal{U}_{c p}=\left\{u: \mathbb{R} \rightarrow U \subset \mathbb{R}^{m}: u\right.$ constante por partes $\}$, onde $U$ é um conjunto compacto.

Observe que, tanto controles em malha aberta quanto perturbações que dependem do tempo, podem ser expressas da mesma maneira, como funções $u$ pertencentes a algum conjunto de funções admissíveis $\mathcal{U}$.

Ainda outra forma de interpretar (1) é como a modelagem de um sistema dinâmico nominal levando em conta incertezas nos parâmetros. Aqui, os campos $X_{1}, \ldots, X_{m}$ modelam a "estrutura" das incertezas nos parâmetros e o conjunto $U$ determina a limitação destas incertezas.

Neste trabalho, restringiremos nosso estudo à classe de sistemas de controle chamados Sistemas de Controle Afins. Esta é uma classe bastante geral e importante de sistemas de controle não lineares que incluem os sistemas lineares e também os bilineares. Os sistemas de controle afins são caracterizados por terem sua equação de evolução com a estrutura de (1), isto é, os controles $u_{i}$ aparecem multiplicando os campos $X_{i}$. Esta já é uma classe bastante geral de sistemas de controle e os conjuntos de acessibilidade em tempo $t$ de tais sistemas apresentam boas propriedades topológicas que serão utilizadas no capítulo 3 na análise do comportamento assintótico de tais conjuntos. A compacidade dos conjuntos de acessibilidade pode ser demonstrada para sistema de controle afins sob hipóteses bem razoáveis, mas não vale para sistemas mais gerais, mesmo que as variáveis de controle fiquem restritas a subconjuntos compactos. Um contra-exemplo pode ser encontrado em [25], 
pág. 121. Além disso, usaremos fortemente a estrutura afim de (1) para a construção de um sistema dinâmico topológico, chamado Sistema Dinâmico de Controle, que associaremos ao sistema de controle (1) no capítulo 2.

Observemos que, no caso da teoria de controle, a propriedade de controlabilidade tem uma relação muito íntima com recorrência de sistemas dinâmicos (de fato, é um tipo de recorrência). Podemos, então, esperar uma relação entre propriedades assintóticas do sistema de controle e controlabilidade. Para formalizar esta relação, Colonius e Kliemann [10] introduziram o conceito de sistema dinâmico de controle associado ao sistema de controle e, junto com este conceito, introduziram os conceitos de Conjuntos Controláveis e Conjuntos Controláveis por Cadeias, que refletem um certo tipo de recorrência.

O sistema dinâmico de controle é um fluxo produto antissimétrico (Skew Product Flow) no espaço estendido $\mathcal{U} \times M$, onde $\mathcal{U}$ é o espaço dos controles admissíveis considerado com a topologia fraca*. O programa perseguido foi fazer corresponder aos conjuntos controláveis em $M$ conjuntos recorrentes em $\mathcal{U} \times M$ do fluxo de controle. Assim, a decomposição do espaço de estados em conjuntos controláveis estaria associada a decomposições do sistema dinâmico em $\mathcal{U} \times M$. Nosso objetivo inicial foi estudar as condições gerais em que isto pode ser feito.

A ferramenta básica utilizada neste trabalho é a integração dos conceitos e idéias de sistemas dinâmicos topológicos e teoria do controle. Existem várias maneiras de relacionar as duas áreas. Neste trabalho, o vínculo entre sistemas dinâmicos e teoria do controle será feito primeiramente através de uma certa generalização do conceito de sistema dinâmico que considera aplicações multivaloradas na variedade de estados $M$ do sistema, aplicações estas que associam um ponto $x \in M$ e um tempo $t \in \mathbb{R}$ com um subconjunto $A \subset M$ ao invés de um único ponto, como acontece nos sistemas dinâmicos clássicos. Com essa generalização, os conceitos de periodicidade e recorrência em sistemas dinâmicos se traduzem em conceitos de controlabilidade e controlabilidade aproximada em sistemas de controle. Esta relação é desenvolvida com certo detalhe na seção 1.3 e no capítulo 3 deste trabalho.

Outra maneira que usaremos para relacionar sistemas dinâmicos e sistemas de controle, é aquela introduzida por Kliemann e Colonius [10] que consiste em associar um sistema dinâmico topológico (com unicidade), de dimensão infinita, em $\mathcal{U} \times M$, ao sistema de controle, onde $\mathcal{U}$ é o conjunto dos controles (ou ruídos) admissíveis e $M$ é a variedade de estados do sistema de controle. Isto será feito no capítulo 4 e resultará em relações entre periodicidade e transitividade em $\mathcal{U} \times M$ e controlabilidade e controlabilidade 
aproximada em $M$.

Vale observar que a relação entre Sistemas Dinâmicos e Sistemas de Controle não se resume à tradução técnica dos resultados de uma área para outra, mas sim, dentre outros méritos, o intercâmbio entre as duas áreas proporciona para os sistemas dinâmicos, um maior esclarecimento no estudo das perturbações de tais sistemas e, para os sistemas de controle, ajuda a esclarecer o comportamento assintótico dos conjuntos de acessibilidade.

Muitos problemas importantes em análise e projetos de sistemas de controle traduzem-se em questões sobre o comportamento assintótico dos conjuntos de acessibilidade. Como um exemplo típico, considere o seguinte sistema de controle

$$
\dot{x}=f(x, u, w),
$$

onde dois tipos de entradas afetam o comportamento do sistema. A variável de entrada $u$, corresponde ao controle propriamente dito do sistema, o qual podemos interferir diretamente. A variável $w$ modela uma perturbação ou um ruído que influencia o sistema mas que não temos controle direto sobre ele. Suponhamos que algum controle por retro-alimentação $u=g(x)$ é usado com o objetivo de dirigir certos estados do sistema para um subconjunto $F$ da variedade de estados $M$. O sistema em malha fechada se escreve, portanto, como

$$
\dot{x}=f(x, g(x), w)
$$

Se estivermos interessados em saber o comportamento deste sistema para uma determinada condição inicial $x_{0}$ após transcorrido um período grande de tempo, teremos que levar em conta todas as possíveis perturbações admissíveis do sistema, isto é, teremos de estudar o comportamento assintótico dos conjuntos de acessibilidade de (2).

Em [45] há uma compilação de vários trabalhos que utilizam conjuntos de acessibilidade para tratar problemas de controle em sistemas biológicos e econômicos. 


\section{Capítulo 1}

\section{Preliminares}

O objetivo deste capítulo é fixar a notação utilizada neste trabalho e provar que os conjuntos de acessibilidade dos sistemas de controle afins variam continuamente com o tempo, propriedade esta que será fundamental para os resultados do próximo capítulo.

Na seção 1.3 definiremos os Conjuntos de Controlabilidade Total, que é o conceito central desta tese.

\subsection{O Sistema de Controle}

Consideraremos a seguinte classe de sistemas de controle:

$$
\dot{x}(t)=X_{0}(x(t))+\sum_{i=1}^{m} u_{i}(t) X_{i}(x(t))
$$

$u \in \mathcal{U}=\left\{u: \mathbb{R} \rightarrow \mathbb{R}^{m}\right.$ mensurável $: u(t) \in U$ quase sempre $\}$

em uma variedade $M$ de classe $C^{\infty}$ de dimensão finita, onde $X_{0}, \ldots, X_{m}$ são campos de vetores de classe $C^{\infty}$ em $M$ e $U \subset \mathbb{R}^{m}$ é um conjunto compacto e convexo. Sistemas deste tipo são chamados de sistemas de controle afins.

Neste trabalho estudaremos algumas questões relacionadas com o comportamento assintótico do sistema. Portanto, assumiremos que, para cada $u \in \mathcal{U}$ e cada $x \in M$, a equação (1.1) possua sua única solução que começa no ponto $x \in M$, definida para todo $t \in \mathbb{R}$. É bastante conhecido que estas hipóteses se cumprem para o caso em que $M$ é uma variedade compacta. Além disso, elas também estão satisfeitas para famílias de sistemas importantes como os clássicos sistemas lineares e os sistemas de controles invariantes 
a esquerda em grupos de Lie em seus espaços homogêneos. Veja [24] e o capítulo 6 de [25].

As soluções do sistema de controle (1.1) serão denotadas por $\varphi(t, x, u)$, isto é,

$$
\varphi(t, x, u) \text { satisfaz a equação }(1.1) \text { e vale } \varphi(0, x, u)=x .
$$

Chamaremos as soluções $\varphi$ de trajetórias do sistema de controle.

A seguir, listaremos algumas propriedades do conjunto dos controles admissíveis $\mathcal{U}$ e das trajetórias de um sistema de controle que serão freqüentemente usadas neste trabalho. Estas propriedades são de fácil verificação.

1. Invariância de $\mathcal{U}$ por translações no tempo: Dados $u \in \mathcal{U}$ e $s \in \mathbb{R}$, o controle $u(\cdot+s)$ definido por

$$
u(\cdot+s)(t)=u(t+s), t \in \mathbb{R}
$$

pertence a $\mathcal{U}$.

2. Fechamento de $\mathcal{U}$ em relação a operação de concatenação: Dados $u, v \in$ $\mathcal{U}$ e $s \in \mathbb{R}$, o controle $u \wedge_{s} v$ definido por

$$
u \wedge_{s} v(t)=\left\{\begin{array}{ll}
v(t) & \text { se } t \leq s \\
u(t-s) & \text { se } t>s
\end{array},\right.
$$

pertence a $\mathcal{U}$. O controle $u \wedge_{s} v$ é chamado de concatenação de $u$ e $v$.

3. As trajetórias de um sistema de controle satisfazem a seguinte propriedade, conhecida como propriedade do cociclo: dados um controle $u \mathrm{e}$ tempos $t, s \in \mathbb{R}$, temos que,

$$
\varphi(s+t, x, u(\cdot))=\varphi(t, \varphi(s, x, u(\cdot)), u(\cdot+s)),
$$

isto é, a trajetória que começa em $x$ usando o controle $u$ coincide no tempo $s+t$ com a trajetória que começa em $\varphi(s, x, u)$ usando o controle $u(\cdot+s)$ no tempo $t$.

Para um uso da propriedade do cociclo no estudo de equações diferenciais ordinárias não autônomas, veja [42]. 


\subsection{Conjuntos de Acessibilidade e Controla- bilidade}

Dados um sistema de controle, um ponto $x \in M$ e um tempo $t>0$ definimos os conjuntos

$$
\begin{aligned}
& \mathcal{O}_{t}^{+}(x):=\{y \in M ; y=\varphi(t, x, u) \text { para algum } u \in \mathcal{U}\} \\
& \mathcal{O}_{t}^{-}(x):=\{y \in M ; x=\varphi(t, y, u) \text { para algum } u \in \mathcal{U}\}
\end{aligned}
$$

que serão chamados, respectivamente, de conjunto de acessibilidade de $x \mathrm{em}$ tempo $t$ e conjunto controlável a $x$ em tempo $t$. Dado $T \geq 0$, definimos ainda os conjuntos

$$
\begin{array}{ll}
\mathcal{O}_{>T}^{+}(x)=\bigcup_{t \in(T, \infty)} \mathcal{O}_{t}^{+}(x), & \mathcal{O}_{>T}^{-}(x)=\bigcup_{t \in(T, \infty)} \mathcal{O}_{t}^{-}(x), \\
\mathcal{O}_{\leq T}^{+}(x)=\bigcup_{t \in(0, T]} \mathcal{O}_{t}^{+}(x), & \mathcal{O}_{\leq T}^{-}(x)=\bigcup_{t \in(0, T]} \mathcal{O}_{t}^{-}(x) .
\end{array}
$$

Os conjuntos $\mathcal{O}_{>0}^{+}(x)$ e $\mathcal{O}_{>0}^{-}(x)$ serão chamados, respectivamente, de órbita positiva de $x$ e órbita negativa de $x$.

Observação 1.1 Note que $x \in \mathcal{O}_{>T}^{+}(y)$ se, e somente se, $y \in \mathcal{O}_{>T}^{-}(x)$.

O conjunto $\mathcal{O}_{\leq T}^{+}(x)$ nada mais é do que o conjunto de estados para os quais é possível levar o ponto $x$ por uma trajetória admissível do sistema em tempo $\leq T$ e o conjunto $\mathcal{O}_{\leq T}^{-}(x)$ representa os estados que podem ser levados até $x$ em tempo $\leq T$. Se o conjunto $U$ consiste apenas de um ponto, então as órbitas são as soluções de uma única equação diferencial ordinária e, neste caso, coincidem com a noção de órbita de um sistema dinâmico.

As órbitas positivas e negativas de um sistema de controle satisfazem a seguinte regra de transitividade:

Lema 1.1 Para quaisquer pontos $x, y, z \in M$ e $T \geq 0$ temos:

1. $S e y \in \mathcal{O}_{>T}^{+}(x)$ e $z \in \mathcal{O}_{>T}^{+}(y)$, então $z \in \mathcal{O}_{>T}^{+}(x)$.

2. $S$ Se $y \in \mathcal{O}_{>T}^{-}(x)$ e $z \in \mathcal{O}_{>T}^{-}(y)$, então $z \in \mathcal{O}_{>T}^{-}(x)$. 
Demonstração. Se $y \in \mathcal{O}_{>T}^{+}(x)$ e $z \in \mathcal{O}_{>T}^{+}(y)$, então existem $u_{1}, u_{2} \in \mathcal{U}$ e $t_{1}, t_{2}>T$, tais que $\varphi\left(t_{1}, x, u_{1}\right)=y$ e $\varphi\left(t_{2}, y, u_{2}\right)=z$. Se usarmos o controle concatenado

$$
v(t)=\left\{\begin{array}{ll}
u_{1}(t) & \text { se } t \leq t_{1} \\
u_{2}\left(t-t_{1}\right) & \text { se } t>t_{1}
\end{array},\right.
$$

decorre da propriedade do cociclo que

$$
\varphi\left(t_{1}+t_{2}, x, v\right)=\varphi\left(t_{2}, \varphi\left(t_{1}, x, u_{1}\right), v\left(\cdot+t_{1}\right)\right)=\varphi\left(t_{2}, y, u_{2}\right)=z
$$

isto é, $z \in \mathcal{O}_{>T}^{+}(x)$. Analogamente mostra-se a afirmação sobre as órbitas negativas.

Os conjuntos de acessibilidade de um ponto $x$, podem ser pensados como a imagem de uma aplicação

$$
\mathcal{O}^{+}: \mathbb{R}^{+} \times M \rightarrow \operatorname{comp}(M),
$$

onde $\operatorname{comp}(M)$ é o conjunto formado por todos os subconjuntos compactos de $M$.

$O$ fato de os conjuntos de acessibilidade em tempo $t$ serem conjuntos compactos, decorre da dependência contínua das soluções $\varphi$ de (1.1) tanto em relação às condições iniciais como em relação aos controles $u \in \mathcal{U}$. No corolário 1.5 será apresentada uma demonstração deste fato.

Uma topologia no conjunto dos controles admissíveis $\mathcal{U}$ que resulta na continuidade (uniforme em intervalos compactos de tempo) das soluções $\varphi(t, x, \cdot)$ em relação aos controles $u$ é a topologia fraca*.

Mais precisamente, o espaço $\mathcal{U}$ dos controles admissíveis pode ser visto como um subconjunto de $L^{\infty}\left(\mathbb{R}, \mathbb{R}^{m}\right)=\left\{u: \mathbb{R} \rightarrow \mathbb{R}^{m}\right.$ essencialmente limitadas $\}$ que coincide com $\left(L^{1}\left(\mathbb{R}, \mathbb{R}^{m}\right)\right)^{*}$, o dual do espaço das funções integráveis de $\mathbb{R}$ em $\mathbb{R}^{m}$. Para maiores detalhes consulte [8]. Desta forma, $\mathcal{U}$ resulta em um espaço topológico compacto com a topologia fraca*, como mostram os lemas 1.2 e 1.3. Os três lemas seguintes compõe um resultado que está publicado em [15].

Lema 1.2 O conjunto $\mathcal{U}$ é fechado na topologia fraca* de $L^{\infty}\left(\mathbb{R}, \mathbb{R}^{m}\right)$. junto

Demonstração. Dado um intervalo compacto $I \subset \mathbb{R}$, considere o con-

$$
\mathcal{U}_{/ I}=\{u / I: u \in \mathcal{U}\} \subset L^{2}\left(I, \mathbb{R}^{m}\right),
$$

onde $u / I$ denota a restrição do controle $u$ ao intervalo $I$. 
O conjunto $\mathcal{U}_{/ I}$ é fechado e convexo na topologia da norma de $L^{2}\left(I, \mathbb{R}^{m}\right)$, pois, dada uma sequência $\left(u_{n}\right)$ de pontos de $\mathcal{U}_{/ I}$ que converge para $u \in$ $L^{2}\left(I, \mathbb{R}^{m}\right)$, existe uma subsequência de $\left(u_{n}\right)$ que converge pontualmente quase sempre para $u$, o que, dada a compacidade de $U$, mostra que $u(t) \in U$ quase sempre, ou seja, $u \in \mathcal{U}_{/ I}$. A convexidade de $\mathcal{U}_{/ I}$ decorre imediatamente da convexidade de $U$.

Todo conjunto fechado e convexo de um espaço localmente convexo é fracamente fechado (teorema 13, pág. 422 de [19]) e, portanto, $\mathcal{U}_{/ I}$ é fechado na topologia fraca de $L^{2}\left(I, \mathbb{R}^{m}\right)$. Como $L^{2}\left(I, \mathbb{R}^{m}\right)$ é reflexivo, $\mathcal{U}_{/ I}$ é fraca*fechado.

Considere agora uma sequência $\left(u_{n}\right)$ de pontos de $\mathcal{U}$ que converge para $u$ na topologia fraca* de $L^{\infty}\left(\mathbb{R}, \mathbb{R}^{m}\right)$. Assim

$$
\int_{\mathbb{R}}\left\langle y(t), u_{n}(t)\right\rangle d t \rightarrow \int_{\mathbb{R}}\langle y(t), u(t)\rangle d t \text { quando } n \rightarrow \infty
$$

para todo $y \in L^{1}\left(\mathbb{R}, \mathbb{R}^{m}\right)$. Portanto

$$
\int_{I}\left\langle y(t), u_{n}(t)\right\rangle d t \rightarrow \int_{I}\langle y(t), u(t)\rangle d t \text { quando } n \rightarrow \infty
$$

para todo $y \in L^{2}\left(I, \mathbb{R}^{m}\right)$, já que $L^{2}\left(I, \mathbb{R}^{m}\right) \subset L^{1}\left(I, \mathbb{R}^{m}\right)$, ou seja, $u_{n} \stackrel{\text { fraca }^{*}}{\rightarrow} u$ em $L^{2}\left(I, \mathbb{R}^{m}\right)$.

Como $\left(u_{n} / I\right)$ é uma sequência de pontos do conjunto fracamente compacto $\mathcal{U}_{/ I}$, temos que $u \in \mathcal{U}_{/ I}$.

$O$ intervalo $I$ pode ser qualquer intervalo compacto de $\mathbb{R}$, o que mostra que $u(t) \in U$ para quase todo $t \in \mathbb{R}$, isto é, $u \in \mathcal{U}$. Portanto $\mathcal{U}$ é fechado na topologia fraca*.

Lema $1.3 O$ conjunto $\mathcal{U}$ é compacto e metrizável na topologia fraca* de $L^{\infty}\left(\mathbb{R}, \mathbb{R}^{m}\right)$ e a métrica é dada por

$$
d_{\mathcal{U}}(u, v)=\sum_{n=1}^{\infty} \frac{1}{2^{n}} \frac{\left|\int_{\mathbb{R}}\left\langle u(t)-v(t), x_{n}(t)\right\rangle d t\right|}{1+\left|\int_{\mathbb{R}}\left\langle u(t)-v(t), x_{n}(t)\right\rangle d t\right|},
$$

onde $\left\{x_{n}, n \in \mathbb{N}\right\}$ é um conjunto denso e enumerável de $L^{1}\left(\mathbb{R}, \mathbb{R}^{\infty}\right)$ e $\langle\cdot, \cdot\rangle$ denota um produto interno de $\mathbb{R}^{m}$. Com esta métrica, $\mathcal{U}$ resulta num espaço métrico completo compacto e separável. 
Demonstração. Pelo teorema de Alaoglu, a bola unitária no espaço dual de um espaço separável é compacta na topologia fraca*. Além disso, uma métrica-é dada por (1.5) (teorema 3, pág. $434[19])$. Como $L^{\infty}\left(\mathbb{R}, \mathbb{R}^{m}\right)=$ $\left(L^{1}\left(\mathbb{R}, \mathbb{R}^{m}\right)\right)^{*}$, e $L^{1}\left(\mathbb{R}, \mathbb{R}^{m}\right)$ é separável, $L^{\infty}\left(\mathbb{R}, \mathbb{R}^{m}\right)$ possui as bolas de raio finito compactas na topologia fraca*.

Pelo lema $1.2, \mathcal{U}$ é um conjunto fraca*-fechado e como $U$ é limitado, $\mathcal{U}$ está contido em uma bola de raio finito de $L^{\infty}\left(\mathbb{R}, \mathbb{R}^{m}\right)$, que é fraca*-compacta. Logo, $\mathcal{U}$ também é fraca*-compacto. Assim $\mathcal{U}$ é um espaço métrico compacto e, portanto, completo e separável [20].

Considerando $\mathcal{U}$ com a topologia descrita no lema 1.3, podemos demonstrar que as trajetórias $\varphi(t, x, u)$ de um sistema de controle afim variam continuamente, tanto em relação às condições iniciais, quanto em relação aos controles $u$. Esta é a razão para adotarmos a topologia fraca* em $\mathcal{U}$. Como corolário, resultará a continuidade dos conjuntos de acessibilidade em relação ao tempo e a compacidade de $\mathcal{O}_{t}^{+}(x)$.

Lema $1.4 A$ aplicação $\varphi: \mathbb{R} \times M \times \mathcal{U} \rightarrow M$, onde $\varphi(t, x, u)$ é como em (1.2), é uma aplicação contínua.

Demonstração. Considere as sequências $t^{n} \rightarrow t^{0}$ em $\mathbb{R}, u^{n} \rightarrow u^{0}$ em $\mathcal{U}$ e $x^{n} \rightarrow x^{0}$ em $M$. De maneira abreviada vamos escrever $\varphi^{n}(t)=$ $\varphi\left(t, x^{n}, u^{n}\right), t \in \mathbb{R}, n=0,1, \ldots$ Vamos mostrar que $\varphi^{n}\left(t^{n}\right) \rightarrow \varphi^{0}\left(t^{0}\right)$. Em coordenadas locais podemos escrever

$$
\left|\varphi^{n}\left(t^{n}\right)-\varphi^{0}\left(t^{0}\right)\right| \leq\left|\varphi^{n}\left(t^{n}\right)-\varphi^{n}\left(t^{0}\right)\right|+\left|\varphi^{n}\left(t^{0}\right)-\varphi^{0}\left(t^{0}\right)\right| .
$$

A primeira parcela desta soma vai à zero, já que $t^{n} \rightarrow t^{0} \mathrm{e}$, quanto a segunda, temos a seguinte estimativa:

$$
\begin{gathered}
\left|\varphi^{n}\left(t^{0}\right)-\varphi^{0}\left(t^{0}\right)\right| \leq\left|x^{n}-x^{0}\right| \\
+\left|\int_{0}^{t^{0}}\left[X_{0}\left(\varphi^{n}(\tau)\right)-X_{0}\left(\varphi^{0}(\tau)\right)\right] d \tau\right| \\
+\left|\int_{0}^{t^{0}} \sum_{i=1}^{m} u_{i}^{n}(\tau)\left[X_{i}\left(\varphi^{n}(\tau)\right)-X_{i}\left(\varphi^{0}(\tau)\right)\right] d \tau\right| \\
+\left|\int_{0}^{t^{0}} \sum_{i=1}^{m}\left[u_{i}^{n}-u_{i}^{0}\right](\tau) X_{i}\left(\varphi^{0}(\tau)\right) d \tau\right| .
\end{gathered}
$$


Aqui, a primeira e a última parcela desta soma convergem para zero pois $x^{n} \rightarrow x^{0}$ e $u^{n} \rightarrow u^{0}$ fracamente. A segunda e a terceira parcela podem ser limitadas por

$$
c_{1} \int_{0}^{t^{0}}\left|\varphi^{n}(\tau)-\varphi^{0}(\tau)\right| d \tau
$$

onde $c_{1}$ depende do diâmetro do conjunto $U$ e das constantes de Lipschitz dos campos $X_{0}, \ldots, X_{m}$.

Assim,

$$
\left|\varphi^{n}\left(t^{0}\right)-\varphi^{0}\left(t^{0}\right)\right| \leq \varepsilon_{n}+c_{1} \int_{0}^{t^{0}}\left|\varphi^{n}(\tau)-\varphi^{0}(\tau)\right| d \tau, \text { onde } \varepsilon_{n} \rightarrow 0 .
$$

Aplicando agora o lema de Gronwall podemos concluir que

$$
\left|\varphi^{n}\left(t^{n}\right)-\varphi^{0}\left(t^{0}\right)\right| \leq \varepsilon_{n} e^{t^{0} c_{1}}
$$

isto é, $\varphi^{n}\left(t^{n}, x^{n}, u^{n}\right) \rightarrow \varphi^{0}\left(t^{0}, x^{0}, u^{0}\right)$.

O conjunto comp $(M)$ também resulta em um espaço métrico se usarmos a métrica de Hausdorff ([27], pág. 131) na qual a distância entre dois conjuntos $A, B \in \operatorname{comp}(M)$ é dada por

$$
\mathrm{d}(A, B)=\max \left\{d_{1}(A, B), d_{1}(B, A)\right\}
$$

onde, se $d(\cdot, \cdot)$ é a distância em $M$,

$$
d(x, Y)=\inf _{y \in Y} d(x, y) \quad \text { e } \quad d_{1}(X, Y)=\sup _{x \in X} d(x, Y) .
$$

A seguir, demonstraremos que os conjuntos $\mathcal{O}_{t}^{+}(x)$ são compactos e, em relação a métrica de Hausdorff, a aplicação $\mathcal{O}^{+}$definida em (1.4) é contínua.

\section{Corolário 1.5 :}

1. Os conjuntos $\mathcal{O}_{t}^{+}(x)$ são compactos seja qual for o $\operatorname{par}(t, x) \in \mathbb{R}^{+} \times M$.

2. A aplicação $\mathcal{O}^{+}$definida em 1.4 é contínua na métrica de Hausdorff.

Demonstração. Conforme o lema 1.3, o conjunto $\mathcal{U}$ é compacto. Temos

$$
\mathcal{O}_{t}^{+}(x)=\varphi(t, x, \mathcal{U})
$$


ou seja, $\mathcal{O}_{t}^{+}(x)$ é a imagem de um conjunto compacto por uma aplicação contínua.

Para o ítem 2, dados $x_{0} \in M, t_{0}>0$ e $\varepsilon>0$, existe $\delta>0$ tal que, se $d\left(x, x_{0}\right)<\delta$ e $\left|t-t_{0}\right|<\delta$ então, para qualquer $u \in \mathcal{U}$ temos

$$
d\left(\varphi(t, x, u), \varphi\left(t_{0}, x_{0}, u\right)\right)<\varepsilon,
$$

o que implica que $\mathcal{O}_{t}^{+}(x) \subset S_{\varepsilon}\left(\mathcal{O}_{t_{0}}^{+}\left(x_{0}\right)\right)$, onde $S_{\varepsilon}\left(\mathcal{O}_{t_{0}}^{+}\left(x_{0}\right)\right)=\{y \in M$ : $\left.d\left(y, \mathcal{O}_{t_{0}}^{+}\left(x_{0}\right)\right)<\varepsilon\right\}$. O mesmo argumento mostra que $\mathcal{O}_{t_{0}}^{+}\left(x_{0}\right) \subset S_{\varepsilon}\left(\mathcal{O}_{t}^{+}(x)\right)$.

\subsection{Conjuntos de Controlabilidade Total}

Um sistema de controle é dito completamente controlável se, para quaisquer pontos $x, y \in M$ temos $y \in \mathcal{O}_{>0}^{+}(x)$. Para sistemas de controle da forma (1.1), não podemos, em geral, esperar controlabilidade total, mesmo que o conjunto $U$ seja ilimitado. Vamos, portanto, considerar o conjunto $\mathcal{P}=\{x \in$ $\left.M: x \in \mathcal{O}_{>1}^{+}(x)\right\}$, isto é, o conjunto dos pontos pelos quais passa pelo menos uma trajetória periódica do sistema (1.1). Note que

$$
\mathcal{P}=\left\{x \in M: x \in \mathcal{O}_{>1}^{+}(x)\right\}=\left\{x \in M: x \in \mathcal{O}_{>\delta}^{+}(x), \forall \delta>0\right\} .
$$

Veremos que o conjunto $\mathcal{P}$ divide-se em subconjuntos nos quais o sistema é completamente controlável. Para definirmos precisamente estes subconjuntos, diremos que dois pontos de $\mathcal{P}$ são equivalentes se eles pertencem a uma mesma trajetória periódica. Em outras palavras, vamos definir uma relação de equivalência $\mathfrak{o}$ em $\mathcal{P}$ da seguinte forma:

Definição 1.1 Dados dois pontos $x, y \in \mathcal{P}$, diremos que $x$ o y se, e somente se, $x \in \mathcal{O}_{>0}^{+}(y)$ e $y \in \mathcal{O}_{>0}^{+}(x)$.

A relação o é realmente uma relação de equivalência pois $x \mathfrak{o} x$ já que $x \in \mathcal{P}$ e $x$ o $y$ implica $y o x$ pela própria definição 1.1. A transitividade desta relação decorre da transitividade das órbitas positivas, conforme o lema 1.1. O conjunto $\mathcal{P}$ fica, portanto, particionado em conjuntos dois a dois disjuntos que são as classes de equivalência da relação 0 . Para cada $x \in \mathcal{P}$, denotaremos a classe de equivalência da relação o a qual $x$ faz parte por $\mathcal{H}_{x}$. 
Resulta que, para $x \in \mathcal{P}$, o conjunto $\mathcal{H}_{x}$ é completamente controlável, no sentido que $\mathcal{H}_{x}$ é o maior conjunto que contém $x$ tal que, tomados dois quaisquer de seus pontos, $y$ e $z$ por exemplo, podemos encontrar controles $u_{1}, u_{2} \in \mathcal{U}$ e tempos $t_{1}, t_{2}>0$ tais que $\varphi\left(t_{1}, y, u_{1}\right)=z$ e $\varphi\left(t_{2}, z, u_{2}\right)=y$. Por isso chamaremos as classes de equivalência da relação o de Conjuntos de Controlabilidade Total.

Esta definição dos conjuntos de controlabilidade total é devida a Emilio Roxin, que apresentou suas propriedades fundamentais em [34]. Paralelamente, T.L. Vincent e J.E. Gayek fizeram um estudo específico de tais conjuntos em [21] onde chamaram os conjuntos de controlabilidade total de 'maneuverable set'. Vários autores consideraram tais conjuntos de maneira implícita em seus trabalhos. Podemos citar, por exemplo, [31], [26]. Em um contexto mais amplo, onde é estudada a ação de um semi-grupo topológico $S$ sobre a variedade $M, \mathrm{~L}$. A. San Martin [comunicação pessoal], considerou as classes de equivalência da relação a para a ação do semi-grupo $S$. Neste contexto, os conjuntos de controlabilidade total coincidem com o núcleo de outro conjunto de recorrência, os conjuntos controláveis, que estudaremos mais adiante. Para mais detalhes, ver [San Martin].

Nesta tese, estudaremos algumas propriedades topológicas destes conjuntos para algumas classes de sistemas de controle, bem como propriedades de recorrência que aparecem quando usamos alguns conceitos de sistemas dinâmicos no estudo de sistemas de controle. Abaixo estão listadas algumas das propriedades elementares dos Conjuntos de Controlabilidade Total.

Proposição 1.6 Dado $x \in \mathcal{P}$ temos que $\mathcal{H}_{x}=\mathcal{O}_{>T}^{+}(x) \cap \mathcal{O}_{>T}^{-}(x)$ para qualquer $T \in[0, \infty)$.

Demonstração. Seja $y \in \mathcal{H}_{x}$. Neste caso, $y \in \mathcal{O}_{>0}^{+}(x)$ e $x \in \mathcal{O}_{>0}^{+}(y)$ o que é equivalente a $y \in \mathcal{O}_{>0}^{+}(x)$ e $y \in \mathcal{O}_{>0}^{-}(x)$. Existem, portanto, controles $u_{1}, u_{2} \in \mathcal{U}$ e tempos $t_{1}, t_{2}>0$ tais que $y=\varphi\left(t_{1}, x, u_{1}\right)$ e $x=\varphi\left(t_{2}, y, u_{2}\right)$. Isto mostra que $y \in \mathcal{O}_{>0}^{+}(x) \cap \mathcal{O}_{>0}^{-}(x)$.

Para qualquer $T>0, \exists n \in \mathbb{N}$ tal que $n\left(t_{1}+t_{2}\right)>T$. Denotando a concatenação $u_{1} \wedge_{t_{2}} u_{2}$ por $v$, temos $x=\varphi\left(t_{1}+t_{2}, x, v\right)$, o que implica que

$$
x=\varphi\left(n\left(t_{1}+t_{2}\right), x, n v\right)
$$

onde $n v$ denota a concatenação $v \wedge_{t_{1}+t_{2}} \ldots \wedge_{t_{1}+t_{2}} v$ do controle $v$ consigo mesmo $n$ vezes e, portanto,

$$
y=\varphi\left(t_{1}+n\left(t_{1}+t_{2}\right), x, u_{1} \wedge_{n\left(t_{1}+t_{2}\right)} n v\right)
$$


o que mostra que $y \in \mathcal{O}_{>T}^{+}(x)$. A mesma construção mostra que $x \in \mathcal{O}_{>T}^{+}(y)$, o que implica que $y \in \mathcal{O}_{>T}^{+}(x) \cap \mathcal{O}_{>T}^{-}(x)$.

A recíproca é imediata, a partir da definição da relação $o$, já que se $y \in$ $\mathcal{O}_{>T}^{+}(x) \cap \mathcal{O}_{>T}^{-}(x)$ então $y \mathfrak{o} x$, isto é, $y \in \mathcal{H}_{x}$.

No sistema afim (1.1), se o campo $X_{0}$ se anula em um ponto $x_{0} \in M$ e o conjunto $U$ contiver a origem, teremos $x-0 \in \mathcal{P}$ e, portanto, $\mathcal{H}_{x_{0}} \neq \emptyset$. É como se o ponto fixo $x_{0}$ se expandisse no conjunto de trajetórias periódicas $\mathcal{H}_{x_{0}}$. Dependendo dos campos $X_{i}, i=1, \ldots, m$ e do diâmetro do conjunto $U$, o conjunto $\mathcal{H}_{x_{0}}$ pode ser toda uma vizinhança de $x_{0}$ ou ser simplesmente igual a $\left\{x_{0}\right\}$. Para sistemas bilineares, isto é, sistemas da forma (1.1) com os campos $X_{i}, i=0,1, \ldots, m$ todos lineares, se o diâmetro de $U$ for suficientemente pequeno, teremos, conforme resultados do capítulo 5 , que $\mathcal{H}_{0}=\{0\}$. Também no capítulo 5, mostraremos que para uma certa classe de perturbações de sistemas lineares hiperbólicos, teremos que $\mathcal{H}_{0}$ sempre será um conjunto aberto (teorema 4.11).

Observação 1.2 Note que, para qualquer $T \geq 0, \mathcal{O}_{>T}^{+}(x) \cap \mathcal{O}_{>T}^{-}(x) \neq \emptyset$ se e somente se $x \in \mathcal{P}$. Podemos então estender a relação o para todos os pontos da variedade $M$ associando, para cada $x \in M$ a classe de equivalência $\mathcal{H}_{x}=\mathcal{O}_{>0}^{+}(x) \cap \mathcal{O}_{>0}^{-}(x)$. Os pontos $x \in M$ tais que $\mathcal{O}_{>0}^{+}(x) \cap \mathcal{O}_{>0}^{-}(x)=$ $\emptyset$ serão chamados de pontos transientes que correspondem exatamente ao complementar do conjunto $\mathcal{P}$.

Proposição 1.7 Para todo $x \in M$, a classe de equivalência $\mathcal{H}_{x}$ é um conjunto conexo por caminhos.

Demonstração. Se $\mathcal{H}_{x}=\emptyset$, não há nada para demonstrar. Seja $x \in M$ tal que $\mathcal{H}_{x} \neq \emptyset$, e sejam $x_{1}, x_{2} \in \mathcal{H}_{x}$.

Como $x_{1} \mathfrak{o} x_{2}$, existe $u \in \mathcal{U}$ e $T>0$ tal que $\varphi\left(T, x_{1}, u\right)=x_{2}$. O caminho $\varphi\left([0, T], x_{1}, u\right)$ está todo contido em $\mathcal{H}_{x}$ pois, para todo $s \in(0, T)$, temos que $x_{0}=\varphi\left(s, x_{1}, u\right) \in \mathcal{H}_{x}$. De fato, por hipótese, temos $x_{1} \in \mathcal{O}_{>0}^{+}(x)$ e, como $x_{0} \in \mathcal{O}_{s}^{+}\left(x_{1}\right)$, resulta que $x_{0} \in \mathcal{O}_{>0}^{+}(x)$. Além disso, $x_{2} \in \mathcal{O}_{>0}^{-}(x)$, e como $x_{0} \in \mathcal{O}_{T-s}^{-}\left(x_{2}\right)$, temos $x_{0} \in \mathcal{O}_{>0}^{-}(x)$. Assim, $x_{0} \in \mathcal{O}_{>0}^{+}(x) \cap \mathcal{O}_{>0}^{-}(x)=\mathcal{H}_{x}$.

Observação 1.3 A demonstração da proposição 1.7 mostra que, se uma trajetória do sistema (1.1) começa e termina em um dado conjunto de controlabilidade total, então ela deve estar toda contida neste conjunto, isto é, se $\varphi(\cdot, x, u)$ é tal que existem $t_{1}, t_{2} \in \mathbb{R}, t_{2}>t_{1}$ e $y \in M \operatorname{com} \varphi\left(t_{1}, x, u\right) \in \mathcal{H}_{y}$ e $\varphi\left(t_{2}, x, u\right) \in \mathcal{H}_{y}$ então $\varphi(t, x, u) \in \mathcal{H}_{y}$ para todo $t \in\left[t_{1}, t_{2}\right]$. 
Proposição 1.8 Se para algum $x \in \mathcal{P}, \mathcal{H}_{x}$ possui um ponto interior, então $\mathcal{H}_{x}$ é um conjunto aberto de $M$.

Demonstração. Sejam $y$ um ponto interior de $\mathcal{H}_{x}$ e $z$ um ponto qualquer de $\mathcal{H}_{x}$. Vamos mostrar que $z$ também é um ponto interior de $\mathcal{H}_{x}$. Por hipótese, existe um conjunto aberto $V_{y}$ tal que $y \in V_{y} \subset \mathcal{H}_{x}$ e um controle $u \in \mathcal{U}$ de forma que $y=\varphi\left(t_{1}, z, u\right)$ e $z=\varphi\left(t_{2}, z, u\right)$ para tempos $t_{2}>t_{1}>0$ convenientes.

As aplicações $\varphi\left(t_{2}-t_{1}, \cdot, u\left(\cdot+t_{1}\right)\right): M \rightarrow M$ bem como $\left(\varphi\left(t_{1}, \cdot, u\right)\right)^{-1}: M \rightarrow$ $M$ são homeomorfismos e, portanto, os conjuntos

$$
V_{z}:=\varphi\left(t_{2}-t_{1}, \cdot, u\left(\cdot+t_{1}\right)\right)\left(V_{y}\right)
$$

bem como

$$
W_{z}:=\left(\varphi\left(t_{1}, \cdot, u\right)\right)^{-1}\left(V_{y}\right)
$$

são abertos que contém o ponto $z$. Agora, para qualquer ponto $z^{\prime} \in V_{z} \cap W_{z}$, existem pontos $y_{1}^{\prime}, y_{2}^{\prime} \in V_{y}$ tais que $\varphi\left(t_{2}-t_{1}, y_{1}^{\prime}, u\left(\cdot+t_{1}\right)\right)=z^{\prime}$ e $\varphi\left(t_{1}, z^{\prime}, u\right)=$ $y_{2}^{\prime}$.

Escolhendo o controle

$$
v(t)= \begin{cases}u\left(t+t_{1}\right) & \text { para } t<t_{2}-t_{1} \\ u(t) & \text { para } t \geq t_{2}-t_{1}\end{cases}
$$

temos que

$$
\varphi\left(0, y_{1}^{\prime}, v\right)=y_{1}^{\prime} \text { e } \varphi\left(t_{2}, y_{1}^{\prime}, v\right)=y_{2}^{\prime}
$$

ambos pertencem a $V_{y} \subset \mathcal{H}_{x}$ e, portanto, $\varphi\left(s, y_{1}^{\prime}, v\right) \in \mathcal{H}_{x} \forall s \in\left[0, t_{2}\right]$, conforme observação (1.3), o que implica que $z^{\prime} \in \mathcal{H}_{x}$. Logo, $V_{z} \cap W_{z} \subset \mathcal{H}_{x}$, isto é, $\mathcal{H}_{x}$ é aberto.

Exemplo 1.1 Considere em $\mathbb{R}$ o sistema

$$
\dot{x}=x+u \quad, \quad u(t) \in[-1,1] .
$$

Observe que se $x>1, \dot{x}$ é positivo independentemente de qual controle usamos. Portanto, todas as trajetórias que começam em $x$ se deslocam no sentido positivo, o que implica que $\mathcal{O}_{>0}^{+}(x) \subset(x, \infty)$ (neste caso, na verdade, temos $\mathcal{O}_{>0}^{+}(x)=(x, \infty)$ ). Além disso, nenhum ponto de $(x, \infty)$ pertence a $\mathcal{O}_{>0}^{-}(x)$ pois, se $x>1$, é impossivel voltar para $x$ a partir de pontos maiores do que $x$. Logo, se $x>1$ temos $\mathcal{H}_{x}=\emptyset$. Da mesma forma, se $x<-1$, todas 
as possíveis trajetórias que começam em $x$ estão indo no sentido decrescente e portanto $\mathcal{O}_{>0}^{+}(x) \cap \mathcal{O}_{>0}^{-}(x)=\emptyset$. Para $x=1$ temos que para $u \equiv-1 x$ é ponto fixo de $(1.6)$ e portanto $1 \in \mathcal{H}_{1}$. Para todos os outros controles admissiveis, as trajetórias positivas começando em 1 serão crescentes, isto é, $\mathcal{O}_{>0}^{+}(1) \subset[1, \infty)$. Porém, se $y>1$, não existe trajetória que, começando em y e usando tempos positivos, passe por $x$, isto é, $\mathcal{O}_{>0}^{-}(1) \subset(-\infty, 1]$. Logo $\mathcal{H}_{1}=\{1\}$. Uma análise similar pode ser feita para $x=-1$ e temos $\mathcal{H}_{-1}=\{-1\}$. Para os pontos $x \in(-1,1)$, o sistema pode mover-se tanto para a direita como para a esquerda e resulta que para estes pontos vale $\mathcal{H}_{x}=(-1,1)$. Neste exemplo existem, portanto, 3 conjuntos de controlabilidade total: $\{-1\},\{1\}$ e $(-1,1)$.

A análise feita neste exemplo pode ser generalizada para todos os sistemas cujo o estado é o conjunto $\mathbb{R}$. Este é o tema da próxima subseção.

\subsubsection{Sistemas de controle em $\mathbb{R}$.}

Devido a peculiar facilidade na análise dos sistemas de controle definidos em $\mathbb{R}$, nesta subseção consideraremos uma classe mais geral de sistemas de controle. Especificamente, vamos considerar sistemas do tipo

$$
\dot{x}=f(x, u)
$$

com $x \in \mathbb{R}$ e $u \in \mathcal{U}=\{u: \mathbb{R} \rightarrow \mathbb{R}$ mensurável : $u(t) \subset U$ quase sempre $\}$, onde $U$ é um intervalo compacto de $\mathbb{R}$ e $f$ é uma função contínua.

Para sistemas deste tipo, o estudo dos conjuntos de controlabilidade total fica bastante facilitado e pode ser feito analisando-se apenas os zeros da função $f(x, u)$.

A proposição abaixo ajuda na compreenção dos conjuntos de controlabilidade total, pois fornece uma maneira simples de analizar tais conjuntos para sistemas de controle em $\mathbb{R}$. Esta proposição é uma adaptação direta de um resultado de [12]. A diferença aqui é que estamos tratando dos conjuntos de controlabilidade total, enquanto que em [12] o contexto é dos conjuntos controláveis, conceito este que trataremos com mais detalhes na seção 3 do capítulo 3 .

Considere os conjuntos

$$
Z=\{x \in \mathbb{R}: \exists u \in U \text { tal que } f(x, u)=0\}
$$




$$
\begin{gathered}
P=\{x \in \mathbb{R}: \exists u \in U \text { tal que } f(x, u)>0\} \\
N=\{x \in \mathbb{R}: \exists u \in U \text { tal que } f(x, u)<0\} \\
Z P=Z \cap P \\
Z N=Z \cap N
\end{gathered}
$$

Proposição 1.9 Para o sistema unidimensional (1.7) temos:

1. O conjunto $\mathcal{P}$ coincide com o conjunto $Z$.

2. Se $x \in(Z-Z P) \cup(Z-Z N)$ então $\mathcal{H}_{x}=x$.

3. Se $x \in Z P \cap Z N$ então $\mathcal{H}_{x}$ é a componente conexo de $Z P \cap Z N$ que contém $x$.

Demonstração. Para a o ítem 1 , se $x \notin Z$, existe $\varepsilon>0$ tal que $(x-$ $\varepsilon, x+\varepsilon) \cap Z=\emptyset$. Se $f(x, u)>0$ para todo par $(x, u) \in(x-\varepsilon, x+\varepsilon) \times U$ temos que as soluções $\varphi(t, x, u)$ de (1.7) são crescentes enquanto $\varphi(t, x, u) \in$ $(x-\varepsilon, x+\varepsilon)$, isto é, $\mathcal{O}_{>0}^{+}(x) \subset(x,+\infty)$. Se $f(x, u)<0$ neste intervalo, então todas as trajetórias que começam em $x$ são decrescentes, o que implica em $\mathcal{O}_{>0}^{-}(x) \subset(-\infty, x)$. Portanto, se $x \notin Z, \mathcal{O}_{>0}^{+}(x) \cap \mathcal{O}_{>0}^{-}(x)=\emptyset$. Por outro lado, se $x \in Z$ então $x$ pode ser pensado como uma órbita periódica (ponto fixo) para algum $u \in U$. Logo, $x \in \mathcal{H}_{x}$.

Para o ítem 2, se $x \in Z-Z P$, então $\mathcal{O}_{>0}^{+}(x) \subset(-\infty, x]$ e $\mathcal{O}_{>0}^{-}(x) \subset[x, \infty)$ o que implica que $\mathcal{H}_{x}=\mathcal{O}_{>0}^{+}(x) \cap \mathcal{O}_{>0}^{-}(x) \subset\{x\}$. Para $x \in Z-Z N$ temos $\mathcal{O}_{>0}^{+}(x) \subset[x, \infty)$ e $\mathcal{O}_{>0}^{-}(x) \subset(-\infty, x]$ e da mesma forma $\mathcal{H}_{x}=\mathcal{O}_{>0}^{+}(x) \cap$ $\mathcal{O}_{>0}^{-}(x) \subset\{x\}$. Usando-se a

primeira parte da demonstração temos $x \in \mathcal{H}_{x}$. Logo, $x=\mathcal{H}_{x}$.

Para o ítem 3 , se $x \in Z P \cap Z N$ definimos:

$$
\begin{aligned}
& p^{+}(x)=\sup \{y \in \mathbb{R}:[x, y) \subset P\} . \\
& n^{+}(x)=\inf \{y \in \mathbb{R}:(y, x] \subset N\} . \\
& p^{-}(x)=\sup \{y \in \mathbb{R}:[x, y) \subset N\} . \\
& n^{-}(x)=\inf \{y \in \mathbb{R}:(y, x] \subset P\} .
\end{aligned}
$$

Mesmo que algumas destas quantidades sejam infinitas, vale que

$$
\mathcal{O}_{>0}^{+}(x)=\left(n^{+}(x), p^{+}(x)\right) \quad \text { e } \quad \mathcal{O}_{>0}^{-}(x)=\left(n^{-}(x), p^{-}(x)\right) .
$$

Denotando por $a=\sup \left\{n^{+}(x), n^{-}(x)\right\}$ e $b=\inf \left\{p^{+}(x), p^{-}(x)\right\}$ temos que $\mathcal{O}_{>0}^{+}(x) \cap \mathcal{O}_{>0}^{-}(x)=(a, b)$, que é justamente a componente conexa de $Z P \cap Z N$ que contém $x$. 
Exemplo 1.2 Considere em $\mathbb{R}$ o sistema

$$
\dot{x}=\operatorname{sen}(x)+u \quad, \quad u(t) \in\left[-\frac{\sqrt{2}}{2}, \frac{\sqrt{2}}{2}\right]
$$

Neste caso,

$$
\begin{array}{ll}
\mathcal{H}_{x}=\emptyset & \text { se } x \notin\left[n \pi-\frac{\pi}{4}, n \pi+\frac{\pi}{4}\right] . \\
\mathcal{H}_{x}=x & \text { se } x=n \pi-\frac{\pi}{4} \text { ou } x=n \pi+\frac{\pi}{4} . \\
\mathcal{H}_{x}=\left(n \pi-\frac{\pi}{4}, n \pi+\frac{\pi}{4}\right) & \text { se } x \in\left(n \pi-\frac{\pi}{4}, n \pi+\frac{\pi}{4}\right) .
\end{array}
$$

onde $n \in \mathbb{Z}$. Ver figura 1.1

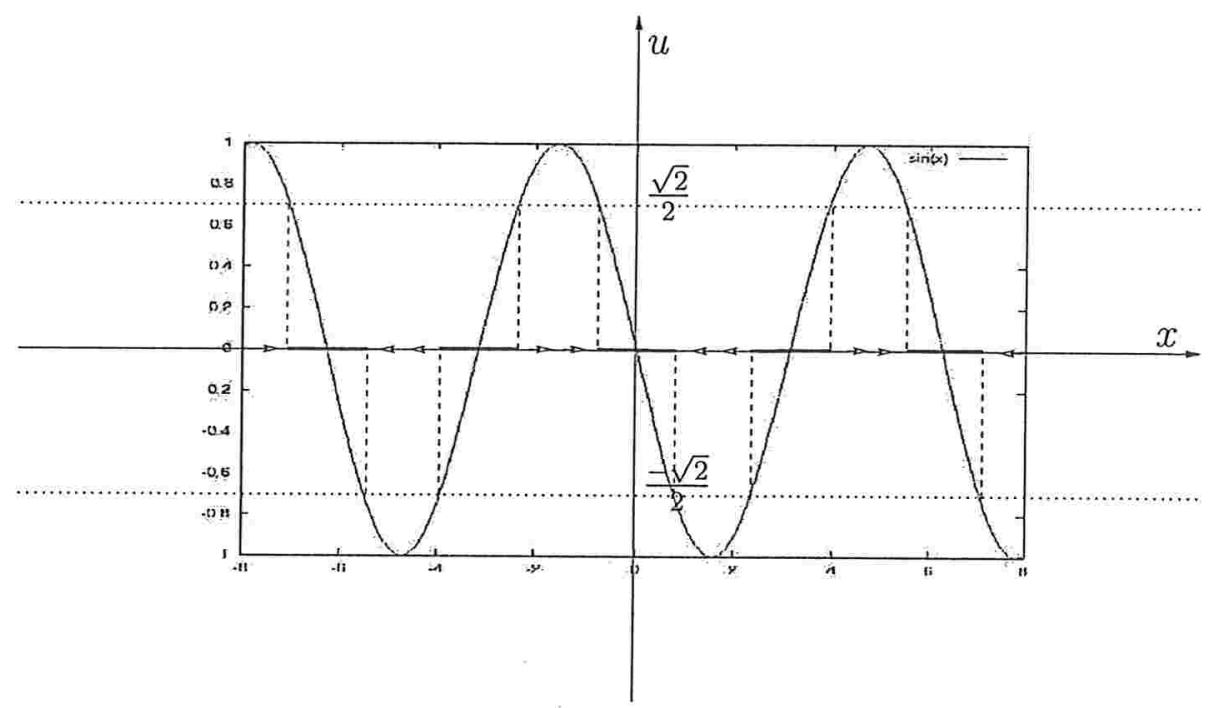

Figura 1.1: Conjuntos de Controlabilidade Total para o exemplo 1.2

Vale observar que para sistemas de controle definidos em $S^{1}$ pode acontecer que o conjunto de zeros $Z$ seja um subconjunto próprio dos conjuntos de controlabilidade total como mostra o exemplo abaixo.

Exemplo 1.3 Considere em $M=S^{1}$ o sistema

$$
\dot{\theta}=\operatorname{sen}^{2}\left(\frac{\theta}{2}\right)+u \cos ^{2}\left(\frac{\theta}{2}\right), u(t) \in[-1,1] .
$$

Neste exemplo, para todo $\theta$ temos $\mathcal{H}_{\theta}=S^{1} \simeq(-\pi, \pi]$, basta usarmos $u \equiv 1$. No entanto, $Z=[-2 \arctan (1), 2 \arctan (1)]=\left[-\frac{\pi}{2}, \frac{\pi}{2}\right]$. 


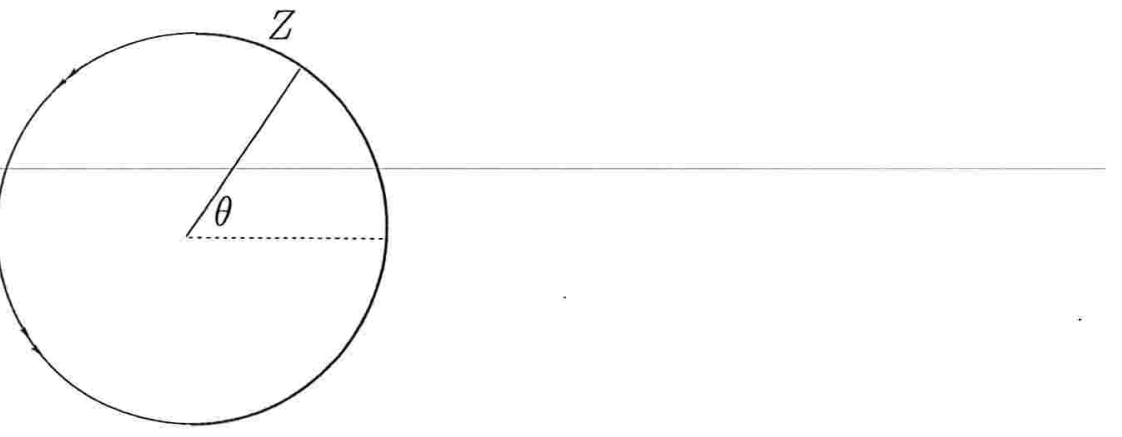

Figura 1.2: Um exemplo onde o Conjunto de Controlabilidade Total é diferente do conjunto $Z$.

Vale observar que, para sistemas de dimensão maior que 1, o problema de determinar os conjuntos de controlabilidade é muito mais difícil. No livro de E. Roxin [33] há alguns exemplos de conjuntos de controlabilidade total para sistemas de controle lineares em $\mathbb{R}^{2}$. No capítulo 5 desta tese, faremos um estudo mais detalhado para sistemas lineares e perturbações de sistemas lineares de controle em $\mathbb{R}^{n}$. Mostraremos que, sob certas condições, há um único conjunto de controlabilidade total. 


\section{Capítulo 2}

\section{Comportamento Assintótico de $\mathcal{O}_{t}^{+}(x)$.}

Neste capítulo estudaremos algumas propriedades assintóticas dos conjuntos de acessibilidade $\mathcal{O}_{t}^{+}(y)$, para pontos $y$ pertencentes a algum conjunto de controlabilidade total. Veremos que estes conjuntos convergem, segundo a métrica de Hausdorff, para conjuntos compactos $\mathbf{R}_{y}^{\infty}$ que satisfazem algumas propriedades dinâmicas notáveis. Por exemplo, estes conjuntos são periódicos, no sentido que existe um número $\tau>0$ tal que $\mathcal{O}_{\tau}^{+}\left(\mathbf{R}_{y}^{\infty}\right)=\mathbf{R}_{y}^{\infty}$. Além disso, o $\omega$-limite de qualquer dos pontos $y \in \mathcal{H}_{x}$ (ver definição 2.1), é sempre igual ao conjunto $\overline{\mathcal{O}_{>0}^{+}\left(\mathbf{R}_{x}^{\infty}\right)}$.

Os resultados apresentados na próxima seção podem ser enunciados sob um ponto de vista axiomático, apenas supondo que os conjuntos de acessibilidade $\mathcal{O}_{t}^{+}(x)$ são compactos e variam continuamente com o tempo (segundo a métrica de Hausdorff), independentemente da estrutura da classe de sistemas de controle considerada. No entanto, neste trabalho, ficaremos restritos apenas à classe dos sistemas afins, pois usaremos fortemente esta estrutura no capítulo 4, quando associaremos um fluxo em $\mathcal{U} \times M$ ao sistema de controle que nos permitirá relacionar propriedades de controlabilidade do sistema de controle com propriedades de recorrência deste fluxo.

Em toda a próxima seção, as convergências de conjuntos serão entendidas segundo a métrica de Hausdorff no espaço métrico comp $(M)$. 


\subsection{Conjuntos de Controlabilidade Total e o Comportamento Assintótico dos Conjun- tos de Acessibilidade.}

O seguinte lema, adaptado de [31], é a peça fundamental para os resultados que seguem.

Lema 2.1 Se $A \subset M$ é um conjunto compacto tal que $\mathcal{O}_{>0}^{+}(A)$ é limitado e $A \subset \mathcal{O}_{\tau}^{+}(A)$ para algum $\tau>0$, então existe um conjunto compacto $R_{A}^{\infty}$ tal que :

1. $\mathcal{O}_{t+\tau}^{+}\left(R_{A}^{\infty}\right)=\mathcal{O}_{t}^{+}\left(R_{A}^{\infty}\right)$ para qualquer $t \geq 0$.

2. $\mathcal{O}_{t}^{+}(A) \rightarrow \mathcal{O}_{t}^{+}\left(R_{A}^{\infty}\right)$ quando $t \rightarrow \infty$.

Demonstração. Como $A \subset \mathcal{O}_{\tau}^{+}(A)$ temos que $\mathcal{O}_{k \tau+t}^{+}(A) \subset \mathcal{O}_{(k+1) \tau+t}^{+}(A)$ para todo $t \in[0, \tau]$. Fixado $t \in[0, \tau]$, os conjuntos $\mathcal{O}_{k \tau+t}^{+}(A)$ são compactos para todo $k \in \mathbb{N}$. Como, $\forall k, \mathcal{O}_{k \tau+t}^{+}(A) \subset \mathcal{O}_{>0}^{+}(A)$ e $\mathcal{O}_{>0}^{+}(A)$ é limitado, resulta que a sequência de compactos $\left(\mathcal{O}_{k \tau+t}^{+}(A)\right)_{k}$ possui uma subsequência convergente

$$
\mathcal{O}_{k_{i} \tau+t}^{+}(A) \rightarrow R_{A, t}^{\infty}
$$

No entanto, $\mathcal{O}_{k \tau+t}^{+}(A) \subset \mathcal{O}_{(k+1) \tau+t}^{+}(A)$ implica em que toda a sequência $\left(\mathcal{O}_{k \tau+t}^{+}(A)\right)_{k}$ converge para o compacto $R_{A, t}^{\infty}$.

Note que $\mathcal{O}_{(k+1) \tau+0}^{+}(A)=\mathcal{O}_{k \tau+\tau}^{+}(A)$ o que implica $R_{A, 0}^{\infty}=R_{A, \tau}^{\infty}$. Os ítens 1 e 2 resultam deste fato se denotarmos $R_{A}^{\infty}:=R_{A, 0}^{\infty}$ e mostrarmos que $R_{A, t}^{\infty}=$ $\mathcal{O}_{t}^{+}\left(R_{A}^{\infty}\right)$. Para isso, observe que, para qualquer $t \in[0, \tau]$, a continuidade das soluções $\varphi$ (lema 1.4) em relação a $x$ e $u$, implica em

$$
R_{A, t}^{\infty}=\mathcal{O}_{t}^{+}\left(R_{A}^{\infty}\right) \text {. }
$$

De fato, se $\bar{z} \in \mathcal{O}_{t}^{+}\left(R_{A}^{\infty}\right)$, então $\bar{z}=\varphi(t, z, u)$, para algum $u \in \mathcal{U}$, onde

$$
z=\lim _{k \rightarrow \infty} \varphi\left(k \tau, x, u_{k}\right) \text { para algum } x \in A .
$$

Assim, $\bar{z}=\lim _{k \rightarrow \infty} \varphi\left(t, \varphi\left(k \tau, x, u_{k}\right), u\right)=\lim _{k \rightarrow \infty} \varphi\left(t+k \tau, x, u \wedge_{k \tau} u_{k}\right) \in R_{A, t}^{\infty}$.

Para mostrar a inclusão contrária, tome $\bar{z} \in R_{A, t}^{\infty}$. Então $\bar{z}=\lim \varphi\left(t+k \tau, x, u_{k}\right)$ para algum $x \in A$ e alguma sequência $\left(u_{k}\right)$ de $\mathcal{U}$. Considere $z=\lim \varphi(k \tau, x, u) \in$ $R_{A}^{\infty}$ e a sequência $\left(v_{k}\right)$ em $\mathcal{U}$ definida por

$$
v_{k}(s)=u_{k}(s+k \tau), \quad s \in \mathbb{R} .
$$


Como $\mathcal{U}$ é compacto, a sequência $\left(v_{k}\right)$ possui uma subsequência convergente, que denotaremos novamente por $\left(v_{k}\right)$, isto é,

$$
\lim _{k \rightarrow \infty} v_{k}=\bar{v} \in \mathcal{U}
$$

Assim, usando novamente o lema 1.4,

$\bar{z}=\lim _{k \rightarrow \infty} \varphi\left(t+k \tau, x, u_{k}\right)=\lim _{k \rightarrow \infty} \varphi\left(t, \varphi\left(k \tau, x, u_{k}\right), v_{k}\right)=\varphi(t, z, \bar{v}) \in \mathcal{O}_{t}^{+}\left(R_{A}^{\infty}\right)$.

Lema 2.2 Dado $A \subset M$ compacto tal que $\mathcal{O}_{\tau}^{+}(A)=A$ para algum $\tau>0$. Se $\tau^{\prime} \in[0, \infty)$ é tal que $A \subset \mathcal{O}_{\tau^{\prime}}^{+}(A)$, então $A=\mathcal{O}_{\tau^{\prime}}^{+}(A)$.

Demonstração. Primeiramente, suponhamos que exista um período $\tau_{0}>0$ que é o menor período de todos, isto é, $\mathcal{O}_{\tau_{0}}^{+}(A)=A$ e se $\mathcal{O}_{\tau^{\prime \prime}}^{+}(A)=A$ para $0 \leq \tau^{\prime \prime}<\tau_{0}$, então $\tau^{\prime \prime}=0$.

Podemos escrever $\tau^{\prime}=k \tau_{0}+\tau^{\prime \prime}$ para algum $k \in\{0,1,2, \ldots\}$ e algum $\tau^{\prime \prime} \in\left[0, \tau_{0}\right)$. Desta forma,

$$
A \subset \mathcal{O}_{\tau^{\prime}}^{+}(A)=\mathcal{O}_{k \tau_{0}+\tau^{\prime \prime}}^{+}(A)=\mathcal{O}_{\tau^{\prime \prime}}^{+}\left(\mathcal{O}_{k \tau_{0}}^{+}(A)\right)=\mathcal{O}_{\tau^{\prime \prime}}^{+}(A)
$$

Pelo lema 2.1, existe um conjunto compacto $R_{A}^{\infty}$ tal que $\mathcal{O}_{\tau^{\prime \prime}}^{+}\left(R_{A}^{\infty}\right)=R_{A}^{\infty}$ e $\mathcal{O}_{t}^{+}(A) \stackrel{t \rightarrow \infty}{\rightarrow} \mathcal{O}_{t}^{+}\left(R_{A}^{\infty}\right)$.

Como $\mathcal{O}_{t}^{+}\left(R_{A}^{\infty}\right)$ e $\mathcal{O}_{t}^{+}(A)$ são ambos periódicos, isto é, $\mathcal{O}_{\tau^{\prime \prime}}^{+}\left(R_{A}^{\infty}\right)=R_{A}^{\infty}$ e $\mathcal{O}_{\tau_{0}}^{+}(A)=A$, resulta que $\mathcal{O}_{t}^{+}(A)=\mathcal{O}_{t}^{+}\left(R_{A}^{\infty}\right), \forall t \geq 0$, ou seja, $A=\mathcal{O}_{\tau^{\prime \prime}}^{+}(A)$ para $\tau^{\prime \prime}<\tau_{0}$, o que, conforme escolha de $\tau_{0}$, implica em $\tau^{\prime \prime}=0$, isto é, $\tau^{\prime}=k \tau_{0}$ e, portanto, $A=\mathcal{O}_{\tau^{\prime}}^{+}(A)$.

Agora, suponhamos que exista uma sequência $\tau_{k} \rightarrow 0, \tau_{k}>0$ tal que $\mathcal{O}_{\tau_{k}}^{+}(A)=A$ para todo $k \in \mathbb{N}$. Neste caso, $A=\mathcal{O}_{n \tau_{k}}^{+}(A) \forall k, n \in \mathbb{N}$. O conjunto $\left\{n \tau_{k} ;(n, k) \in \mathbb{N} \times \mathbb{N}\right\}$ é denso em $\mathbb{R}^{+}$, e como $\mathcal{O}_{t}^{+}(A)$ é contínuo em $t$, resulta que $A=\mathcal{O}_{t}^{+}(A) \forall t \geq 0$. Portanto, vale o lema também neste caso.

Corolário 2.3 Se $A \subset M$ é um conjunto compacto tal que $\mathcal{O}_{\tau}^{+}(A)=A$ para algum $\tau>0$ e existem $\tau_{1}, \tau_{2} \geq 0$ tais que $A \subset \mathcal{O}_{\tau_{1}}^{+}(A)$ e $A \subset \mathcal{O}_{\tau_{2}}^{+}(A)$, então $A=\mathcal{O}_{m \tau_{1}+n \tau_{2}}^{+}(A)$ para quaisquer $m, n \in \mathbb{N}$.

Se $\tau_{1}$ e $\tau_{2}$ são incomensuráveis, então $A=\mathcal{O}_{t}^{+}(A) \forall t \geq 0$. 
Demonstração. $\mathcal{O}_{m \tau_{1}+n \tau_{2}}^{+}(A)=\mathcal{O}_{m \tau_{1}}^{+}\left(\mathcal{O}_{n \tau_{2}}^{+}(A)\right)=\mathcal{O}_{m \tau_{1}}^{+}(A)=A$ e, se $\tau_{1}$ e $\tau_{2}$ são incomensuráveis, então os pontos $m \tau_{1}+n \tau_{2}$ são densos em $\mathbb{R}^{+}$, o que implica em $\mathcal{O}_{t}^{+}(A)=A$ para todo $t \geq 0$.

Teorema 2.4 Se $x \in M$ é tal que $\mathcal{H}_{x} \neq \emptyset$ e se $\mathcal{O}_{>0}^{+}(x)$ é um conjunto limitado, então, para todo $z \in \mathcal{H}_{x}$, existe um conjunto compacto $R_{z}^{\infty}$ tal que:

1. $\mathcal{O}_{t}^{+}(z) \rightarrow \mathcal{O}_{t}^{+}\left(R_{z}^{\infty}\right)$ quando $t \rightarrow \infty$.

2. Existe $\tau>0$ tal que $\mathcal{O}_{t+\tau}^{+}\left(R_{z}^{\infty}\right)=\mathcal{O}_{t}^{+}\left(R_{z}^{\infty}\right) \forall t \geq 0$.

3. Para qualquer $y \in \mathcal{H}_{x}$, existe $t_{1}(y) \geq 0$ tal que $\mathcal{O}_{t}^{+}(y) \rightarrow \mathcal{O}_{t+t_{1}}^{+}\left(R_{z}^{\infty}\right)$ quando $t \rightarrow \infty$.

Demonstração. Se $z \in \mathcal{H}_{x}$, então $z \in \mathcal{O}_{\tau}^{+}(z)$ para algum $\tau>0$ e os ítens 1 e 2 seguem diretamente do lema 2.2.

Para o ítem 3, note que, se $\mathcal{H}_{x}=\{x\}, z=x$ e basta escolher $t_{1}(x)=0$. Caso contrário, se $y \in \mathcal{H}_{x}$ e $y \neq z$, então existem $t_{1}, t_{2}>0$ tais que $y \in \mathcal{O}_{t_{1}}^{+}(z)$ e $z \in \mathcal{O}_{t_{2}}^{+}(y)$. Pelo lema 2.1,

$$
\mathcal{O}_{t}^{+}(z) \stackrel{t \rightarrow \infty}{\rightarrow} \mathcal{O}_{t}^{+}\left(R_{z}^{\infty}\right) \text { e } \mathcal{O}_{t}^{+}(y) \stackrel{t \rightarrow \infty}{\rightarrow} \mathcal{O}_{t}^{+}\left(R_{y}^{\infty}\right)
$$

Como $z \in \mathcal{O}_{t_{2}}^{+}(y)$, vale que $\mathcal{O}_{t}^{+}(z) \subset \mathcal{O}_{t+t_{2}}^{+}(y)$, o que implica em $\mathcal{O}_{t}^{+}\left(R_{z}^{\infty}\right) \subset$ $\mathcal{O}_{t+t_{2}}^{+}\left(R_{y}^{\infty}\right)$. Da mesma forma, $\mathcal{O}_{t}^{+}\left(R_{y}^{\infty}\right) \subset \mathcal{O}_{t+t_{1}}^{+}\left(R_{z}^{\infty}\right)$. Portanto, valendo-se do lema 2.2, temos

$$
\mathcal{O}_{t}^{+}\left(R_{y}^{\infty}\right) \subset \mathcal{O}_{t+t_{1}}^{+}\left(R_{z}^{\infty}\right) \subset \mathcal{O}_{t+t_{1}+t_{2}}^{+}\left(R_{y}^{\infty}\right)=\mathcal{O}_{t}^{+}\left(R_{y}^{\infty}\right)
$$

o que implica em

$$
\mathcal{O}_{t}^{+}\left(R_{y}^{\infty}\right)=\mathcal{O}_{t+t_{1}}^{+}\left(R_{z}^{\infty}\right) \forall t \geq 0
$$

Teorema 2.5 Dado $x \in M$ com $\mathcal{H}_{x} \neq \emptyset$ e $\mathcal{O}_{>0}^{+}(x)$ limitado. Se $z \in \mathcal{H}_{x}$, considerando o conjunto compacto $\mathbf{R}_{c}:=\bigcup_{s \in[0, \tau]} \mathcal{O}_{s}^{+}\left(R_{z}^{\infty}\right)=\mathcal{O}_{>0}^{+}\left(R_{z}^{\infty}\right)$, temos:

1. $\mathcal{O}_{t}^{+}\left(\overline{\mathcal{H}_{x}}\right) \subset \mathbf{R}_{c} \forall t \geq 0$.

2. $\mathcal{O}_{t}^{+}\left(\overline{\mathcal{H}_{x}}\right) \rightarrow \mathbf{R}_{c}$ quando $t \rightarrow \infty$. 
3. $\mathrm{R}_{c}=\overline{\mathcal{O}_{>0}^{+}(z)}$.

Se existe $(y, u) \in \mathcal{H}_{x} \times U$ tal que $X_{0}(y)=-\sum_{i=1}^{m} u_{i} X_{i}(y)$ então

4. $\mathcal{O}_{t}^{+}\left(R_{z}^{\infty}\right) \equiv R_{z}^{\infty}=\mathbf{R}_{c} \forall t \geq 0$.

Demonstração. Vamos mostrar que $\mathcal{O}_{t}^{+}(z) \subset \mathbf{R}_{c} \forall z \in \mathcal{H}_{x}$ e $\forall t \geq 0$. Suponhamos, por absurdo, que, para algum $z^{\prime} \in \mathcal{H}_{x}$ e algum $t^{\prime} \geq 0$ exista $\bar{z} \in \mathcal{O}_{t^{\prime}}^{+}\left(z^{\prime}\right)$ tal que $\bar{z} \notin \mathbf{R}_{c}$.

Como $z^{\prime} \in \mathcal{H}_{x}$, existe $\tau>0$ tal que $z^{\prime} \in \mathcal{O}_{\tau}^{+}\left(z^{\prime}\right)$ e, portanto, $\forall k \in \mathbb{N}$, $\bar{z} \in \mathcal{O}_{k \tau+t^{\prime}}^{+}\left(z^{\prime}\right)$. No entanto, pelo teorema 2.4, ítem 3, existe $t_{1}(z) \geq 0$ tal que

$$
\mathcal{O}_{k \tau+t^{\prime}}^{+}\left(z^{\prime}\right) \stackrel{t \rightarrow \infty}{\rightarrow} \mathcal{O}_{k \tau+t^{\prime}+t_{1}}^{+}\left(R_{z}^{\infty}\right)
$$

o que contradiz $\bar{z} \notin \mathbf{R}_{c}$. Portanto, $\mathcal{O}_{t}^{+}\left(\mathcal{H}_{x}\right) \subset \mathbf{R}_{c} \forall t \geq 0$, o que, dada a compacidade de $\mathbf{R}_{c}$, mostra o ítem 1 .

Para o ítem 2, primeiramente observe que $\mathcal{H}_{x} \subset \mathcal{O}_{t}^{+}\left(\mathcal{H}_{x}\right) \forall t \geq 0$, pois cada ponto de $\mathcal{H}_{x}$ pertence a pelo menos uma solução periódica do sistema de controle, e esta solução está necessariamente toda contida em $\mathcal{H}_{x}$. Desta forma $\overline{\mathcal{H}_{x}} \subset \mathcal{O}_{t}^{+}\left(\overline{\mathcal{H}_{x}}\right) \forall t \geq 0$, e pelo lema 2.1 ,

$$
\mathcal{O}_{t}^{+}\left(\overline{\mathcal{H}_{x}}\right) \stackrel{t \rightarrow \infty}{\rightarrow} R_{\mathcal{H}_{x}}^{\infty}
$$

que satisfaz

$$
\mathcal{O}_{t}^{+}\left(R_{\mathcal{H}_{x}}^{\infty}\right)=R_{\mathcal{H}_{x}}^{\infty} \forall t \geq 0
$$

Vamos mostrar que $R_{\mathcal{H}_{x}}^{\infty}=\mathbf{R}_{c}$.

Conforme o ítem 1 , temos

$$
\mathcal{O}_{t}^{+}\left(\overline{\mathcal{H}_{x}}\right) \subset \mathbf{R}_{c} \forall t \geq 0,
$$

o que implica em $R_{\mathcal{H}_{x}}^{\infty} \subset \mathbf{R}_{c}$. Agora,

$$
\mathcal{O}_{t}^{+}(z) \subset \mathcal{O}_{t}^{+}\left(\overline{\mathcal{H}_{x}}\right) \forall t \geq 0,
$$

o que implica em

$$
\mathcal{O}_{t}^{+}\left(R_{z}^{\infty}\right) \subset \mathcal{O}_{t}^{+}\left(R_{\mathcal{H}_{x}}^{\infty}\right)=R_{\mathcal{H}_{x}}^{\infty} \forall t \geq 0 \mathrm{e},
$$

portanto, $\mathbf{R}_{c} \subset R_{\mathcal{H}_{x}}^{\infty}$. Logo

$$
\mathcal{O}_{t}^{+}\left(\overline{\mathcal{H}_{x}}\right) \stackrel{t \rightarrow \infty}{\rightarrow} R_{\mathcal{H}_{x}}^{\infty}=\mathrm{R}_{c}
$$


Para o ítem 3, das relações 2.2 e 2.1 temos $\mathcal{O}_{>0}^{+}(z) \subset \mathbf{R}_{c}$ o que implica $\overline{\mathcal{O}_{>0}^{+}(z)} \subset \mathbf{R}_{c}$, já que $\mathbf{R}_{c}$ é compacto. Para a inclusão contrária, seja $y \in \mathbf{R}_{c}$. Então $y \in \mathcal{O}_{s_{0}}^{+}\left(R_{z}^{\infty}\right)$ para algum $s_{0} \in[0, \tau]$, isto é, existe $w \in R_{z}^{\infty}$ tal que $y=\varphi\left(s_{0}, w, v\right)$ para algum $v \in \mathcal{U}$. Pelo ítem 2 do teorema 2.4 temos que

$$
\mathcal{O}_{k \tau}(z) \rightarrow R_{z}^{\infty} \text { quando } k \rightarrow \infty
$$

isto é, existe uma sequência $\left(u_{k}\right)$ em $\mathcal{U}$ tal que $\varphi\left(k \tau, z, u_{k}\right) \rightarrow w$ e, portanto,

$$
\varphi\left(k \tau+s_{0}, z, v \wedge u_{k}\right) \rightarrow \varphi\left(s_{0}, w, v\right)=y .
$$

Logo, $y \in \overline{\mathcal{O}_{>0}^{+}(z)}$ e a igualdade entre os conjuntos fica estabelecida.

Ao estudarmos o comportamento assintótico das trajetórias de um sistema dinâmico, um conjunto que desempenha um papel importante é o $\omega$ limite. A seguir, enunciaremos a definição do $\omega$-limite de um ponto $x$ para sistemas de controle, que é uma generalização direta da definição de $\omega$-limite para sistemas dinâmicos. Ver [34], [3].

Definição 2.1 $O$ w-limite de um ponto $x \in M$ é o conjunto

$$
\Omega(x)=\bigcap_{s>0} \overline{\mathcal{O}_{>s}^{+}(x)}
$$

Este conjunto corresponde aos pontos $y \in M$ tais que existem sequências $t_{i} \rightarrow \infty$ e $u_{i} \in \mathcal{U}$ com $y=\lim \varphi\left(t_{i}, x, u_{i}\right)$.

Na demonstração do teorema 2.5 , podemos ver que todos os pontos de um conjunto de controlabilidade total $\mathcal{H}_{x}$ possuem o mesmo $\omega$-limite. Vamos ressaltar este fato na forma de uma proposição.

Proposição 2.6 Dado um conjunto de controlabilidade total $\mathcal{H}_{x} \neq \emptyset$, temos que para todo $z \in \mathcal{H}_{x}$ vale $\Omega(z)=\mathbf{R}_{c}$, onde $\mathbf{R}_{c}$ é dado pelo teorema 2.5.

Demonstração. $\Omega(z)=\cap_{s>0} \overline{\mathcal{O}_{>s}^{+}(z)} \subset \overline{\mathcal{O}_{>0}^{+}(z)}=R_{c}$. Para a inclusão contrária, se $y \in \mathbf{R}_{c}$, (2.3) mostra que $y \in \Omega(z)$.

Quando for possível encontrar uma trajetória constante $\varphi(t, x, u) \equiv x$ para o sistema de controle, o teorema 2.5 ítem 4, mostra que existe um conjunto invariante $\mathbf{R}_{c}$ para a aplicação $\mathcal{O}^{+}$tal que $\mathcal{O}_{t}^{+}(x) \rightarrow \mathbf{R}_{c}$ ao $t \rightarrow \infty$. O seguinte teorema mostra que o mesmo é verdade se $x \in \operatorname{int} \mathcal{O}_{\tau}^{+}(x)$ para algum $\tau>0$ e $\mathcal{H}_{x} \neq\{x\}$. 
Teorema 2.7 Dado $x \in M$ tal que $\mathcal{H}_{x} \neq \emptyset$ e $\mathcal{H}_{x} \neq\{x\}$, se $\mathcal{O}_{>0}^{+}(x)$ é limitado e existe $z \in \mathcal{H}_{x}$ tal que $\operatorname{int} \mathcal{O}_{\tau}^{+}(z) \cap \mathcal{H}_{x} \neq \emptyset$

então existe um conjunto compacto $\mathbf{R}_{c}$ tal que $\mathcal{O}_{t}^{+}(y) \stackrel{t \rightarrow \infty}{\rightarrow} \mathbf{R}_{c} \forall y \in \mathcal{H}_{x}$ e $\mathcal{O}_{t}^{+}\left(\mathbf{R}_{c}\right)=\mathbf{R}_{c} \forall t \geq 0$.

Demonstração. Seja $\bar{z} \in \operatorname{int} \mathcal{O}_{\tau}^{+}(z) \cap \mathcal{H}_{x}$. Como $\bar{z} \in \mathcal{H}_{x}$, temos que $z \in \mathcal{O}_{t_{1}}^{+}(\bar{z})$ para algum $t_{1}>0$ e, portanto, $z \in \mathcal{O}_{t_{1}}^{+}\left(\operatorname{int} \mathcal{O}_{\tau}^{+}(z)\right)=\operatorname{int} \mathcal{O}_{t_{1}+\tau}^{+}(z)$. Assim, existe $\delta>0$ tal que $z \in \mathcal{O}_{t_{1}+\tau+\varepsilon}^{+}(z) \forall \varepsilon \in[0, \delta]$. Pelo corolário 2.3, já que podemos encontrar dois números incomensuráveis da forma $t_{1}+\tau+\varepsilon$ com $\varepsilon \in[0, \delta]$ temos

$$
\mathcal{O}_{t}^{+}(z) \stackrel{t \rightarrow \infty}{\rightarrow} R_{z}^{\infty} \text { tal que } \mathcal{O}_{t}^{+}\left(R_{z}^{\infty}\right)=R_{z}^{\infty} \forall t \geq 0 .
$$

Pelo ítem 3 do teorema 2.4, para todo $y \in \mathcal{H}_{x}, \mathcal{O}_{t}^{+}(y) \stackrel{t \rightarrow \infty}{\rightarrow} R_{z}^{\infty}$. Escolhendo $\mathbf{R}_{c}=R_{z}^{\infty}$, o teorema fica provado.

\subsection{Recorrência}

Considere agora o conjunto

$$
\mathcal{R}:=\left\{x \in M: x \in \overline{\mathcal{O}_{>1}^{+}(x)}\right\}
$$

que será chamado de conjunto dos pontos recorrentes do sistema de controle.

No conjunto $\mathcal{R}$ diremos que dois de seus pontos são equivalentes se pudermos, a partir de qualquer um deles, chegar tão perto quanto queiramos do outro por uma trajetória admissível do sistema (1.1).

Para sermos mais precisos, usaremos a seguinte notação:

$$
x \preccurlyeq y \text { quando } y \in \overline{\mathcal{O}_{>\delta}^{+}(x)} \text { para algum } \delta>0 .
$$

A relação $\preccurlyeq$ é uma relação de ordem parcial em $M$. A transitividade de $\preccurlyeq$ decorre da continuidade das trajetórias do sistema de controle em relação as condições iniciais e está formalizada no lema abaixo.

Lema 2.8 Dados $x, y$ e $z \in M$, se $x \preccurlyeq y$ e $y \preccurlyeq z$ então $x \preccurlyeq z$. 
Demonstração. Temos que existem $\delta_{1}$ e $\delta_{2}$ tais que $y \in \overline{\mathcal{O}_{>\delta_{1}}^{+}(x)}$ e $z \in \overline{\mathcal{O}_{>\delta_{2}}^{+}(y)}$, isto é, existem sequências $\left(t_{i}\right) \in\left(\delta_{1}, \infty\right)$ e $\left(u_{i}\right) \in \mathcal{U}$ tais que $y_{i}=\varphi\left(t_{i}, x, u_{i}\right) \rightarrow y$ e $\left(s_{j}\right) \in\left(\delta_{2}, \infty\right)$ e $v_{j} \in \mathcal{U}$ tais que $z_{j}:=\varphi\left(s_{j}, y, v_{j}\right) \rightarrow$ $z$. Desta forma, para qualquer vizinhança $V$ de $z$ existe $j_{0}$ tal que $z_{j} \in$ $V, \forall j>j_{0}$. Fixado $j>j_{0}$, a aplicação $\varphi\left(s_{j}, \cdot, v_{j}\right): M \rightarrow M$ é um difeomorfismo, o que implica em $W:=\left(\varphi\left(s_{j}, \cdot, v_{j}\right)\right)^{-1}(V)$ ser uma vizinhança de $y$. Para $i_{0}$ suficientemente grande, $y_{i}=\varphi\left(t_{i}, x, u_{i}\right) \in W \quad \forall i>i_{0}$ e, portanto, $\varphi\left(s_{j}, \varphi\left(t_{i}, x, u_{i}\right), v_{j}\right) \in V$, isto é, $\varphi\left(s_{k}+t_{k}, x, v_{k} \wedge_{t_{k}} u_{k}\right) \subset V \quad \forall k>$ $\max \left\{i_{0}, j_{0}\right\}$. Como esta última inclusão vale para qualquer vizinhança $V$ de $z$, temos $z \in \overline{\mathcal{O}_{>\delta_{1}+\delta_{2}}^{+}(x)}$, isto é $x \preccurlyeq z$.

Vamos considerar em $\mathcal{R}$ a relação $\mathfrak{o}_{\sim}$ definida da seguinte forma:

Definição 2.2 Dados $x, y \in \mathcal{R}$, diremos que $x \mathrm{o}_{\sim} y$ se, e somente se,

$$
x \preccurlyeq y \quad \text { e } y \preccurlyeq x .
$$

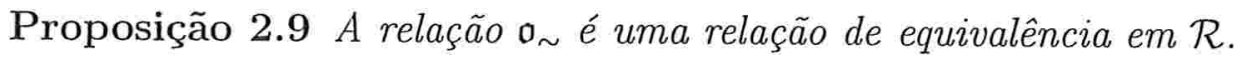

Demonstração. A simetria decorre da própria definição. A transitividade decorre do lema 2.8, e a reflexividade decorre de estarmos considerando apenas pontos de $\mathcal{R}$.

Dado um ponto $x \in \mathcal{R}$, denotaremos a classe de equivalência da relação $\mathfrak{o}_{\sim}$ que contém $x$ por $\mathcal{D}_{x}$ e a chamaremos de Conjunto de Controlabilidade Aproximada.

Observe que na definição do conjunto $\mathcal{R}$, poderíamos exigir apenas que $x \in \overline{\mathcal{O}_{>\delta}^{+}(x)}$ para algum $\delta>0$, isto é, se $\mathcal{R}_{\delta}:=\left\{x \in M: x \in \overline{\mathcal{O}_{>\delta}^{+}(x)}\right\}$ então $\mathcal{R}_{\delta}=\mathcal{R}, \forall \delta>0$. De fato, para $\delta<1, \mathcal{R} \subset \mathcal{R}_{\delta}$ e, se $x \in \mathcal{R}_{\delta}$, então $x \preccurlyeq x 0$ que implica que $x \in \overline{\mathcal{O}_{>n \delta}^{+}(x)}$ para $n \in \mathbb{N}$ e, portanto, $x \in \mathcal{R}$. Para $\delta>10$ raciocínio é análogo.

Proposição 2.10 Um ponto $x \in \mathcal{R}$ se e somente se $x \in \Omega(x)$.

Demonstração. Se $x \in \mathcal{R}$, então $x \in \overline{\mathcal{O}_{>\delta}^{+}(x)}$ para algum $\delta>0$ o que implica que $x \in \overline{\mathcal{O}_{>n \delta}^{+}(x)}$ para todo $n \in \mathbb{N}$ e, portanto, $x \in \Omega(x)$.

A recíproca é imediata.

Por serem classes de equivalência, os conjuntos de controlabilidade aproximada $\mathcal{D}_{x}$ são dois a dois disjuntos. Além disso, satisfazem uma condição de não retorno análoga àquela da observação 1.3 para conjuntos de controlabilidade total, conforme mostra a seguinte proposição. 
Proposição 2.11 Se $y \in \mathcal{D}_{x}$ e existem $T>0$ e $u \in \mathcal{U} \operatorname{com} \varphi(T, y, u) \in \mathcal{D}_{x}$, então $\varphi(t, y, u) \in \mathcal{D}_{x}$ para todo $0 \leq t \leq T$.

Demonstração. Dado $t \in[0, T]$, é claro que $x \preccurlyeq y \preccurlyeq \varphi(t, y, u)$ e, $\varphi(t, y, u) \preccurlyeq \varphi(T, y, u)$. Como $y$ e $\varphi(T, y, u) \in \mathcal{D}_{x}$ temos $\varphi(T, y, u) \preccurlyeq x$ o que resulta, dada a transitividade de $\preccurlyeq$, que $x \preccurlyeq y \preccurlyeq \varphi(t, y, u) \preccurlyeq \varphi(T, y, u) \preccurlyeq x$, isto é, $\varphi(t, y, u) \mathfrak{o}_{\sim} x$.

Como em um sistema de controle existem, em geral, várias trajetórias possíveis começando em $x$, vamos considerar também o comportamento assintótico de cada uma das possíveis trajetórias. Mais especificamente, dados um ponto $x \in M$ e um controle $u \in \mathcal{U}$, vamos definir o conjunto $\omega$-limite do $\operatorname{par}(x, u)$ da seguinte forma:

$$
\omega_{M}(x, u):=\left\{y \in M: \exists t_{i} \rightarrow \infty \text { tal que } \varphi\left(t_{i}, x, u\right) \rightarrow y\right\},
$$

onde $\left(t_{i}\right)_{i}$ é uma sequência monótona de números reais positivos e $\varphi(t, x, u)$ é a solução de (1.1), relativa ao controle $u$ e que começa em $x$.

Considere o conjunto

$$
\mathcal{R}^{\sharp}=\left\{x \in M: \exists u \in \mathcal{U} \operatorname{com} x \in \omega_{M}(x, u)\right\} .
$$

Analisaremos agora a relação entre o conjunto $\mathcal{R}^{\sharp}$ e o conjunto $\mathcal{R}$. Imediatamente podemos dizer que $\mathcal{R}^{\sharp} \subset \mathcal{R}$, já que $\omega_{M}(x, u) \subset \overline{\mathcal{O}_{>1}^{+}(x)}$ para todo $u \in \mathcal{U}$. Se restringirmos a relação de equivalência $\mathfrak{o}_{\sim}$ ao conjunto $\mathcal{R}^{\sharp}$, este fica particionado em classes de equivalência que denotaremos, para $x \in \mathcal{R}^{\sharp}$, por $\mathcal{D}_{x}^{\sharp}$. Na verdade $\mathcal{D}_{x}^{\sharp}=\mathcal{D}_{x} \cap \mathcal{R}^{\sharp}$

O exemplo abaixo mostra que $\mathcal{R}^{\sharp} \neq \mathcal{R}$.

Exemplo 2.1 Considere como espaço de fase $M$ o cilindro $\mathbb{R} / 2 \pi \times \mathbb{R}$ e um sistema de controle da forma

$$
\dot{x}=X_{0}(x(t))+u(t) X_{1}(x(t)) t \in \mathbb{R}, \quad u(t) \in[0,1]
$$

onde

$X_{0}\left(x_{1}, x_{2}\right)=\left(\operatorname{sen}^{2}\left(x_{1}\right)+x_{2}^{2}, x_{2}\left(\operatorname{sen}^{2}\left(x_{1}\right)+x_{2}^{2}\right)\right)$ e $X_{1}\left(x_{1}, x_{2}\right)=\left(0, \alpha\left(x_{1}, x_{2}\right)\right)$.

Aqui, $\alpha$ é uma função $C^{\infty}$, não negativa, definida em $M$, tal que $\alpha\left(x_{1}, x_{2}\right)>0$ se e somente se

$$
\left|\left(x_{1}, x_{2}\right)-\left(\frac{\pi}{2}, 0\right)\right|<\frac{\pi}{4} .
$$


O traço das soluções do sistema sem controle, isto é, com $u \equiv 0$, quando o ponto inicial satisfaz $x_{2}(0) \neq 0$, se consideradas em $\mathbb{R}^{2}$, são dadas pelas exponenciais $x_{2}(0) \exp \left(x_{1}\right)$, pois $\frac{d x_{2}}{d x_{1}}=x_{2}$. Quando $x_{2}(0)=0$, as soluções $\left(x_{1}(t), x_{2}(t)\right)$ satisfazem $\dot{x}_{1}(t) \geq 0$ e $x_{2}(t) \equiv 0 \forall t \geq 0$. Além disso $\dot{x}_{1}(t)=0$ se e somente se $x_{1}(0)=0$ ou $x_{1}(0)=\pi$.

Agora, considere o conjunto $A=\left(0, \frac{3 \pi}{4}\right) \times\{0\} \subset M$. Para o sistema (2.5), vale que $A \subset \mathcal{R}$ e o conjunto $B=\left\{\left(x_{1}, x_{2}\right) \in M ; x_{2}>0\right.$ ou $\left.\frac{3 \pi}{4}<x_{1}<\pi\right\}$ é tal que $B \cap \mathcal{R}=\emptyset$.

No entanto, $\forall x \in A, \forall u \in \mathcal{U}, \exists T>0$ tal que $\varphi(t, x, u) \in B \forall t>T$, o que mostra que, se $x \in A, x \notin \omega(x, u)$ para $u \in \mathcal{U}$. Neste caso $\mathcal{R}^{\sharp} \varsubsetneqq \mathcal{R}$.

O próximo exemplo mostra a diferença entre os conjuntos $\mathcal{H}_{x}$ e os conjuntos $\mathcal{D}_{x}^{\sharp}$ e $\mathcal{D}_{x}$.

Exemplo 2.2 O fluxo irracional no toro $T^{2}$ pode ser pensado como um sistema de controle (onde o conjunto $U=\{0\}$ ). Neste caso temos $\mathcal{H}_{x}=\emptyset e$ $\mathcal{D}_{x}^{\sharp}=\mathcal{D}_{x}=T^{2}$ para todo $x \in T^{2}$.

\subsection{Conjuntos Controláveis.}

Existe na literatura um grande número de trabalhos que estudam as propriedades de certos subconjuntos de $M$ que são chamados, em inglês, de "Control Sets". Ver, por exemplo, [11], [13], [14], [46]. Nas referências do livro [15] há uma lista mais completa.

Neste trabalho, traduziremos o nome Control Set para Conjuntos Controláveis. Na verdade, a definição de control set varia um pouco na literatura. Usaremos aqui a definição dada em [10].

Definição 2.3 Um conjunto $D \subset M$ é chamado de Conjunto Controlável do sistema (1.1) se:

1. Para qualquer ponto $x \in D$, existe uma trajetória $\varphi(\cdot, x, u)$ correspondente a um estado inicial $x$ no tempo $t=0$ e um controle admissível $u \in \mathcal{U}$ tal que, $\varphi(t, x, u) \in D$ para todo $t>0$,

2. $D \subset \overline{\mathcal{O}_{>0}^{+}(x)}$ para qualquer ponto $x \in D$,

3. $D$ é maximal com estas propriedades, isto é, se $D^{\prime} \supset D$ satisfaz 1 e 2 então $D^{\prime}=D$. 
O conceito de conjunto controlável foi introduzido por L. Arnold [2] e suas propriedades foram estudadas por uma série de autores. Recentemente, parte deste material foi sistematizado no livro [15]. Outros autores, como por exemplo, C. J. Braga Barros [5], [6], L. A. B. San Martin [38], [39], [41] P. A. Tonelli [44], generalizaram o conceito de conjunto controlável para o contexto de ações de semigrupos topológicos em variedades, já que, em alguns casos, um sistema de controle induz uma ação de um semigrupo de difeomorfismos na variedade de estados $M$, ver por exemplo Jurdjevic [25].

Nesta seção, vamos estudar a relação entre os conjuntos controláveis, os conjuntos de controlabilidade total, as classes de equivalência $\mathcal{D}_{x}^{\sharp}$ e os conjuntos de controlabilidade aproximada $\mathcal{D}_{x}$.

Proposição 2.12 Qualquer conjunto controlável está contido no conjunto $\mathcal{R}$ e, mais especificamente, em um único conjunto de controlabilidade aproximada.

Demonstração. De fato, dado um ponto $x$ de um conjunto controlável $D$, pela propriedade 1 da definição $2.3, \exists u \in \mathcal{U}$ tal que $\varphi(2, x, u) \in D$. Pela propriedade $2, x \in \overline{\mathcal{O}_{>0}^{+}(\varphi(2, x, u))}$ o que implica em $x \in \overline{\mathcal{O}_{>1}^{+}(x)}$ e, ainda pela propriedade $2, x \mathfrak{o} \sim y$ para todo $y \in D$.

Portanto, se $x$ pertence a algum conjunto controlável $D$, temos $D \subset \mathcal{D}_{x}$.

Proposição 2.13 Toda classe de equivalência $\mathcal{D}_{x}^{\sharp}$ está contida em algum conjunto controlável.

Demonstração. Basta mostrarmos que, para cada $y \in \mathcal{D}_{x}^{\sharp}$, existe $u \in \mathcal{U}$ tal que, para $t \geq 0$, a trajetória $\varphi(t, y, u)$ fica toda contida em um mesmo conjunto controlável, isto é, $\forall t \geq 0, \varphi(t, y, u) \mathfrak{o}_{\sim} x$.

Como $y \in \mathcal{D}_{x}^{\sharp}$, existe $u \in \mathcal{U}$ tal que $y \in \omega_{M}(y, u)$. Vamos mostrar que a trajetória $\varphi(t, y, u)$ fica contida em uma classe de equivalência da relação $o_{\sim}$ para todo $t \geq 0$.

Se $t>0$, vale que $y \preccurlyeq \varphi(t, y, u)$ e $\varphi(t, y, u) \preccurlyeq y$, isto é, $y o_{\sim} \varphi(t, y, u)$. Como $x \mathfrak{o}_{\sim} y$ fica demonstrada a proposição.

Portanto, para $x \in \mathcal{R}^{\sharp}$, temos agora as seguintes inclusões:

$$
\mathcal{H}_{x} \subset \mathcal{D}_{x}^{\sharp} \subset D \subset \mathcal{D}_{x} .
$$

Um conjunto de controlabilidade aproximada $\mathcal{D}_{x}$ não é um conjunto controlável porque, em geral, não podemos garantir a existência, para todo ponto 
$y \in \mathcal{D}_{x}$, de um controle $u \in \mathcal{U}$ que faça com que a trajetória $\varphi(t, y, u)$ permaneça em $\mathcal{D}_{x}$ para todo $t \geq 0$. O próximo exemplo mostra que $D$ pode ser distinto de $\mathcal{D}_{x}$.

Exemplo 2.3 No mesmo sistema do exemplo 2.1 podemos perceber que, em geral, temos $D \varsubsetneqq \mathcal{D}_{x}$. Lá, o conjunto $A=\left(0, \frac{3 \pi}{4}\right) \times\{0\}$ está contido em um único conjunto de controlabilidade aproximada, isto é, $A \subset \mathcal{D}_{\left(\frac{\pi}{2}, 0\right)}$. Note também que o conjunto $B=\left\{\left(x_{1}, x_{2}\right) \in M ; x_{2}>0\right.$ ou $\left.\frac{3 \pi}{4}<x_{1}<\pi\right\}$ é tal que $B \cap \mathcal{R}=\emptyset$. Assim, $\forall x \in A, \forall u \in \mathcal{U}, \exists T>0$ tal que $\varphi(t, x, u) \in$ $B \forall t>T$. Portanto, $\exists x \in \mathcal{D}_{\left(\frac{\pi}{2}, 0\right)}$ tal que, para todo $u \in \mathcal{U}, \varphi(\cdot, x, u) \nsubseteq$ $\mathcal{D}_{\left(\frac{\pi}{2}, 0\right)}$, isto é, $\mathcal{D}_{\left(\frac{\pi}{2}, 0\right)}$ não é um conjunto controlável.

A proposição seguinte mostra que, pelo menos, os conjuntos de controlabilidade aproximada que possuem interior não vazio são conjuntos controláveis.

Proposição 2.14 Se um conjunto de controlabilidade aproximada $\mathcal{D}_{x}$ é tal que int $\mathcal{D}_{x} \neq \emptyset$, então $\mathcal{D}_{x}$ é um conjunto controlável.

Demonstração. Basta mostrarmos que, $\forall y \in \mathcal{D}_{x} \exists v \in \mathcal{U}$ tal que $\varphi(t, y, v) \in \mathcal{D}_{x} \forall t \geq 0$. Seja $z_{0} \in \operatorname{int} \mathcal{D}_{x}$. Como $y \preccurlyeq z_{0}, \exists u_{1} \in \mathcal{U}$ e $t_{1}>$ 0 tal que $z_{1}:=\varphi\left(t_{1}, y, u_{1}\right) \in \operatorname{int} \mathcal{D}_{x}$. Existe $\varepsilon>0$ tal que $z_{2}:=\varphi\left(t_{1}+\right.$ $\left.\varepsilon, y, u_{1}\right) \in \operatorname{int} \mathcal{D}_{x}$. Podemos escolher vizinhanças $V_{z_{1}}$ e $V_{z_{2}}$ dos pontos $z_{1}$ e $z_{2}$ respectivamente, tais que $V_{z_{1}} \subset$ int $\mathcal{D}_{x}, V_{z_{2}} \subset$ int $\mathcal{D}_{x} \mathrm{e}$

$$
\varphi\left(\varepsilon, V_{z_{1}}, \theta_{t_{1}} u_{1}\right) \subset V_{z_{2}} .
$$

Podemos construir o controle $v$ da seguinte forma: Começando em $y$, usamos o controle $u_{1}$ até chegarmos em $z_{2}$. Como $z_{2} \preccurlyeq z_{1}$, existem $t_{2}>0$ e $u_{2} \in \mathcal{U}$ tais que $z_{3}:=\varphi\left(t_{2}, z_{2}, u_{2}\right) \in V_{z_{1}}$. Portanto, devido a relação 2.7, $z_{4}:=\varphi\left(t_{3}, z_{3}, \theta_{t_{1}} u_{1}\right) \in V_{z_{2}}$. Já que $V_{z_{2}} \subset \mathcal{D}_{x}$ existem $u_{3} \in \mathcal{U}$ e $t_{3}>0$ tais que $z_{5}:=\varphi\left(t_{3}, z_{4}, u_{3}\right) \in V_{z_{1}}$.

Repetindo o raciocínio, sempre podemos voltar a $V_{z_{2}}$ usando o controle $\theta_{t_{1}} u_{1}$ e novamente a $V_{z_{1}}$ por algum controle $u_{i}$ já que $V_{z_{2}} \subset \mathcal{D}_{x}$. Desta forma, podemos ir de $V_{z_{1}}$ para $V_{z_{2}}$ infinitas vezes e toda vez que vamos de $V_{z_{1}}$ a $V_{z_{2}}$ gastamos um tempo $\varepsilon$ e, portanto, a trajetória assim construida está definida para todo $t \geq 0$. Dada a condição de não retorno da proposição 2.11 , a parte positiva da trajetória está toda contida em $\mathcal{D}_{x}$, como queríamos demonstrar. 
Capítulo 2. Comportamento Assintótico de $\mathcal{O}_{t}^{+}(x)$.

Definição 2.4 Um sistema de controle é dito localmente acessível em um ponto $x \in M$ quando, para qualquer $T>0$, temos $\operatorname{int}\left(\mathcal{O}_{<T}^{+}(x)\right) \neq \emptyset$.

Quando o sistema de controle é localmente acessível, os conjuntos de controlabilidade aproximada que possuem interior não vazio estão contidos no conjunto $\mathcal{R}^{\sharp}$. Para estabelecermos este resultado, primeiramente demonstraremos o seguinte lema:

Lema 2.15 Dado um conjunto de controlabilidade aproximada $\mathcal{D}_{x}$, se int $\mathcal{D}_{x} \neq$ $\emptyset$ e o sistema é localmente acessível em algum ponto $z \in$ int $\mathcal{D}_{x}$, então $\overline{\operatorname{int} \mathcal{D}_{x}}=\overline{\mathcal{D}_{x}}$.

Demonstração. Vamos mostrar que

$$
\mathcal{D}_{x} \subset \overline{\operatorname{int} \mathcal{D}_{x}} .
$$

Para tanto, basta mostrarmos que, para todo $y \in \mathcal{D}_{x}$ e para qualquer vizinhança $V_{y}$ de $y$, temos $V_{y} \cap \operatorname{int} \mathcal{D}_{x} \neq \emptyset$.

Seja $z:=\varphi(t, y, u) \in \operatorname{int} \mathcal{D}_{x}$ um ponto localmente acessível. Dada a continuidade em relação às condições iniciais, existe uma vizinhança $\widetilde{V}_{y}$ de $y$ tal que $\varphi\left(t, \widetilde{V}_{y}, u\right) \subset \operatorname{int} \mathcal{D}_{x}$. Como $z$ é localmente acessível e $y, z \in \mathcal{D}_{x}$, vale que $y \in \overline{\mathcal{O}_{>0}^{+}(z)}=\overline{\operatorname{int} \mathcal{O}_{>0}^{+}(z)}$ e, portanto, para qualquer vizinhança do ponto y $V_{y} \subset \widetilde{V}_{y}$, teremos int $\mathcal{O}_{>0}^{+}(z) \cap V_{y} \neq \emptyset$.

$\mathrm{O}$ conjunto aberto int $\mathcal{O}_{>0}^{+}(z) \cap V_{y}$ está contido em $\mathcal{D}_{x}$ pois, se $y^{\prime} \in$ int $\mathcal{O}_{>0}^{+}(z) \cap V_{y}$ vale que $z \in \overline{\mathcal{O}_{>0}^{+}(y)}$ e $y^{\prime} \in \mathcal{O}_{>0}^{+}(z)$, o que implica em $y^{\prime} \in \overline{\mathcal{O}_{>0}^{+}(y)}$. Por outro lado, $y \in \overline{\mathcal{O}_{>0}^{+}\left(y^{\prime}\right)}$ pois $y^{\prime} \in V_{y}$ o que implica $z^{\prime}=\varphi\left(t, y^{\prime}, u\right) \in \operatorname{int} \mathcal{D}_{x}$ e, portanto, $y \in \overline{\mathcal{O}_{>0}^{+}\left(z^{\prime}\right)}$. Assim $y \in \overline{\mathcal{O}_{>0}^{+}\left(y^{\prime}\right)}$ o que mostra que $y^{\prime} o_{\sim} y$.

Proposição 2.16 Nas hipóteses do lema 2.15, para todo $y \in \mathcal{D}_{x}$, existe $u \in \mathcal{U}$ tal que $y \in \omega_{M}(y, u)$, isto é, $\mathcal{D}_{x}^{\sharp}=\mathcal{D}_{x}$.

Demonstração. Seja $y \in \mathcal{D}_{x}$. Vamos construir um controle $u \in \mathcal{U}$ tal que $y \in \omega_{M}(y, u)$. Considere uma sequência $\varepsilon_{n} \rightarrow 0$ de números reais positivos e denote por $B(y, \varepsilon)$ a bola aberta de raio $\varepsilon$ em $M$ com centro em $y$. Pelo lema 2.15, $\forall \varepsilon>0, \operatorname{int}\left(B(y, \varepsilon) \cap \mathcal{D}_{x}\right) \neq \emptyset$. Portanto, existem $t_{1}>0$ e $u_{1} \in \mathcal{U}$ tais que $y_{1}:=\varphi\left(t_{1}, y, u_{1}\right) \in \mathcal{D}_{x} \cap B\left(y, \varepsilon_{1}\right)$. Como $y_{1} \in \mathcal{D}_{x}$, existem $t_{2}>0$ e $u_{2} \in \mathcal{U}$ tais que $y_{2}:=\varphi\left(t_{2}, y_{1}, u_{2}\right) \in \mathcal{D}_{x} \cap B\left(y, \varepsilon_{2}\right)$. Seguindo desta 
forma, para todo $n \in \mathbb{N}$, podemos garantir que existem $y_{n} \in \mathcal{D}_{x} \cap B\left(y, \varepsilon_{n}\right)$, $t_{n}>0$ e $u_{n} \in \mathcal{U}$ tais que $\varphi\left(t_{n}, y_{n-1}, u_{n}\right)=y_{n}$.

Definimos então

$$
u(t)=u_{n}\left(t-\sum_{i=0}^{n-1} t_{i}\right) \quad \text { para } \quad t \in\left(\sum_{i=0}^{n-1} t_{i}, \sum_{i=0}^{n} t_{i}\right]
$$

onde $t_{0}=0$. Para $t \leq 0$ podemos definir $u(t)$ de maneira arbitrária e podemos escolher $\left(t_{i}\right)$ tal que $\sum_{i=0}^{\infty} t_{i}=\infty$. Logo, $y \in \omega_{M}(y, u)$.

Note que, dadas as hipótese do lema 2.15 , temos $\mathcal{D}_{x}^{\sharp}=D=\mathcal{D}_{x}$.

Proposição 2.17 Se int $\mathcal{D}_{x} \neq \emptyset$ e o sistema é localmente acessivel em int $\mathcal{D}_{x}$, então int $\mathcal{D}_{x}$ é um conjunto de controlabilidade total.

Demonstração. Basta mostrarmos que, se $y \in \operatorname{int} \mathcal{D}_{x}$ então int $\mathcal{D}_{x}=$ $\mathcal{H}_{y}$.

Seja $y \in \operatorname{int} \mathcal{D}_{x}$. Dada a acessibilidade local em $\operatorname{int} \mathcal{D}_{x}$, para qualquer $z \in \operatorname{int} \mathcal{D}_{x}$ temos que

$$
\operatorname{int} \mathcal{O}^{-}(z) \cap \operatorname{int} \mathcal{D}_{x} \neq \emptyset .
$$

Portanto, existem $t_{1}>0, u_{1} \in \mathcal{U}$ tais que $z^{\prime}:=\varphi\left(t_{1}, y, u_{1}\right) \in \operatorname{int} \mathcal{O}^{-}(z) \cap$ int $\mathcal{D}_{x}$ e existem $t_{2}>0, u_{2} \in \mathcal{U}$ tais que $\varphi\left(t_{2}, z^{\prime}, u_{2}\right)=z$, isto é $y \prec z^{\prime} \prec z$.

Da mesma forma, int $\mathcal{O}^{-}(y) \cap$ int $\mathcal{D}_{x} \neq \emptyset$. Assim, existem $t_{3}>0, u_{3} \in$ $\mathcal{U}$ tais que $y^{\prime}:=\varphi\left(t_{3}, z, u_{3}\right) \in \operatorname{int} \mathcal{O}^{-}(y) \cap \operatorname{int} \mathcal{D}_{x}$ e existem $t_{4}>0, u_{4} \in$ $\mathcal{U}$ tais que $\varphi\left(t_{4}, y^{\prime}, u_{4}\right)=y$, isto é $z \prec y^{\prime} \prec y$. Portanto $y \circ z$ e, dada a generalidade na escolha de $z$, temos $i n t \mathcal{D}_{x} \subset \mathcal{H}_{y}$. A inclusão contrária decorre das inclusões (2.6).

Corolário $2.18 S e \mathcal{H}_{y}$ é um conjunto de controlabilidade total com int $\mathcal{H}_{y} \neq$ $\emptyset$ e o sistema é localmente acessível em $M$, então

$$
\overline{\mathcal{H}_{y}}=\overline{\mathcal{D}_{y}^{\sharp}}=\bar{D}=\overline{\mathcal{D}_{y}} \text {. }
$$

Demonstração. Como $\mathcal{H}_{y} \subset \mathcal{D}_{y}$, temos int $\mathcal{D}_{y} \neq \emptyset$. A proposição 2.17 mostra que int $\mathcal{D}_{y}=\mathcal{H}_{y}$ o que implica $\overline{\mathcal{H}_{y}}=\overline{\mathcal{D}_{y}}$. 


\subsection{Notas}

Em [31], são apresentados resultados análogos aos_da seção 3.1 no contexto de inclusões diferenciais. A principal contribuição deste trabalho reside na descrição dos conjuntos limites $R_{A}^{\infty}$ como sendo os $\omega$-limites dos conjuntos de acessibilidade dos pontos pertencentes aos conjuntos de controlabilidade total.

As seções 3.2 e 3.3 são, basicamente, uma comparação entre os conjuntos controláveis e os conjuntos de controlabilidade aproximada. Essa comparação ajuda a esclarecer o papel das hipóteses de acessibilidade local e int $D \neq \emptyset$ freqüentemente usadas na literatura. 


\section{Capítulo 3}

\section{O Sistema Dinâmico em $\mathcal{U} \times M$.}

Neste capítulo associaremos um fluxo no conjunto $\mathcal{U} \times M$ ao sistema de controle (1.1) e relacionaremos os conjuntos topologicamente mixing deste fluxo (veja definição 3.2), com o fecho dos conjuntos de controlabilidade total $\mathcal{H}_{x}$ em $M$.

A associação dos conjuntos topologicamente mixing do fluxo em $\mathcal{U} \times M$ com os conjuntos controláveis $D$ foi estabelecida em [10], e a contribuição do presente trabalho reside em uma maneira de "levantar" para o produto $\mathcal{U} \times M$ os conjuntos de controlabilidade total, o que permite considerar sistemas de controle não necessariamente localmente acessíveis e evitar hipóteses sobre a topologia dos conjuntos de controlabilidade total. Na seção 3.3, há uma comparação um pouco mais detalhada dos resultados de [10] com os resultados desta tese.

\subsection{O fluxo em $\mathcal{U} \times M$.}

Vamos agora definir um sistema dinâmico em $\mathcal{U} \times M$ associado ao sistema de controle (1.1) que chamaremos de Sistema Dinâmico de Controle.

Para cada $(u, x) \in \mathcal{U} \times M$, seja $\Phi: \mathbb{R} \times \mathcal{U} \times M \rightarrow \mathcal{U} \times M$ a aplicação definida por

$$
\Phi(t,(u, x))=\left(\theta_{t}(u), \varphi(t, x, u)\right)
$$

onde $\theta_{t}: \mathcal{U} \rightarrow \mathcal{U}$ é tal que $\theta_{t}(u)=u(\cdot+t)$, istoé, $\theta_{t}$ é a translação para a direita de tempo $t$ e $\varphi(t, x, u)$ é a solução de (1.1), no tempo $t$, correspondente a ação do controle $u \in \mathcal{U}$. No que segue, será considerada a topologia produto no conjunto $\mathcal{U} \times M$, onde a topologia de $\mathcal{U}$ é a fraca* (lema 1.3). 
O próximo lema está publicado em [10].

Lema 3.1 A aplicação $\Phi$ define um sistema dinâmico contínuo em $\mathcal{U} \times M$.

Demonstração. Primeiramente vamos mostrar que a aplicação $\theta: \mathbb{R} \times$ $\mathcal{U} \rightarrow \mathbb{R} \times \mathcal{U}$ é uma aplicação contínua. Considere as sequências $t^{n} \rightarrow t \in \mathbb{R}$ e $u^{n} \rightarrow u \in \mathcal{U}$. Então, para todo $x \in L^{1}\left(\mathbb{R}, \mathbb{R}^{m}\right)$ temos

$$
\begin{gathered}
\left|\int_{\mathbb{R}}\left\langle u^{n}\left(t^{n}+\tau\right), x(\tau)\right\rangle d \tau-\int_{\mathbb{R}}\langle u(t+\tau), x(\tau)\rangle d \tau\right| \\
\leq\left|\int_{\mathbb{R}}\left\langle u^{n}\left(t^{n}+\tau\right)-u^{n}(t+\tau), x(\tau)\right\rangle d \tau\right| \\
+\left|\int_{\mathbb{R}}\left\langle u^{n}(t+\tau)-u(t+\tau), x(\tau)\right\rangle d \tau\right|= \\
=\left|\int_{\mathbb{R}}\left\langle u^{n}(\tau), x\left(\tau-t^{n}\right)\right\rangle d \tau-\int_{\mathbb{R}}\left\langle u^{n}(\tau), x(\tau-t)\right\rangle d \tau\right| \\
\quad+\left|\int_{\mathbb{R}}\left\langle u^{n}(\tau)-u(\tau), x(\tau-t)\right\rangle d \tau\right| .
\end{gathered}
$$

A segunda parcela desta soma converge para zero pois $u^{n} \rightarrow u$ em $\mathcal{U}$ e a primeira parcela pode ser estimada por

$$
\leq \sup _{w \in U}|w| \int_{\mathbb{R}}\left|x\left(\tau-t^{n}\right)-x(\tau-t)\right| d \tau
$$

Aqui a integral converge a zero quando $t^{n} \rightarrow t$ pois a norma de $L^{1}$ é contínua. Portanto $\theta_{t^{n}}\left(u^{n}\right) \rightarrow \theta_{t}(u)$.

A continuidade da segunda componente de $\Phi$ decorre do lema 1.4.

Para mostrar que $\Phi(t+s,(u, x))=\Phi(t, \Phi(s,(u, x)))$ e $\Phi(0,(u, x))=(u, x)$ basta observar que

$$
\begin{aligned}
\Phi(t+s,(u, x)) & =\left(\theta_{t+s}(u), \varphi(t+s, x, u)\right) \\
& =\left(\theta_{t}\left(\theta_{s}(u)\right), \varphi\left(t, \varphi(s, x, u), \theta_{s}(u)\right)\right)
\end{aligned}
$$

denotando-se $\theta_{s}(u)=v$ e $\varphi(s, x, u)=y$ temos

$$
\begin{aligned}
\left(\theta_{t}\left(\theta_{s}(u)\right), \varphi\left(t, \varphi(s, x, u), \theta_{s}(u)\right)\right) & =\left(\theta_{t}(v), \varphi(t, y, v)\right) \\
& =\Phi(t,(v, y)) \\
& =\Phi(t, \Phi(s,(u, x)))
\end{aligned}
$$


e

$$
\Phi(0,(u, x))=\left(\theta_{0}(u), \varphi(0, x, u)\right)=(u, x)
$$

\subsection{O levantamento de $\mathcal{P}$.}

Vamos considerar em $\mathcal{U} \times M$ o subconjunto $\mathrm{P}$ dos pontos periódicos de $\Phi$, isto é

$$
P:=\{(u, x) \in \mathcal{U} \times M: \exists t>0 \text { tal que } \Phi(t,(u, x))=(u, x)\} .
$$

Naturalmente, o conjunto $\mathbf{P}$ está relacionado com o conjunto $\mathcal{P}$ definido anteriormente. Da mesma maneira que no conjunto $\mathcal{P}$, vamos considerar que dois pontos de $\mathbf{P}$ são equivalentes se eles estiverem em uma mesma trajetória periódica da aplicação $\Phi$. Mais precisamente:

Definição 3.1 Dois pontos $(u, x),(v, y) \in P$ serão considerados equivalentes se existirem $t_{1}, t_{2}>0$ tais que $\Phi\left(t_{1},(u, x)\right)=(v, y)$ e $\Phi\left(t_{2},(v, y)\right)=(u, x)$.

A classe de equivalência desta relação que contém o ponto $(u, x)$ será denotada por $H_{(u, x)}$. O conjunto $H_{(u, x)}$ nada mais é do que a (única) trajetória que contém o ponto $(u, x)$, isto é, estamos individualizando cada uma das trajetórias periódicas que passam pelo ponto $x \in M$.

A fim de sermos mais precisos quanto a relação entre as classes de equivalência do conjunto $\mathcal{P}$ com as do conjunto $\mathbf{P}$ vamos considerar os conjuntos

$$
\mathcal{U}_{x}:=\{u \in \mathcal{U}:(u, x) \in \mathrm{P}\}
$$

e

$$
\mathrm{H}_{x}:=\bigcup_{u \in \mathcal{U}_{x}} H_{(u, x)} .
$$

$\mathrm{O}$ conjunto $\mathrm{H}_{x}$ é um tipo de levantamento para o produto $\mathcal{U} \times M$ do conjunto $\mathcal{H}_{x} \in M$. Note que o conjunto $\mathrm{H}_{x}$ é formado somente por pontos periódicos do fluxo $\Phi$ e qualquer ponto periódico de $\Phi$ está contido no levantamento de algum conjunto de controlabilidade total. Além disso $\pi_{M}\left(\mathbf{H}_{x}\right)=\mathcal{H}_{x}$ e $\Phi\left(\mathbf{H}_{x}\right)=\mathbf{H}_{x}$ 
Outro tipo de levantamento das classes de equivalência $\mathcal{H}_{x}$ para o produto $\mathcal{U} \times M$ é o conjunto

$$
\mathbb{D}_{x}:=\left\{(u, y) \in \mathcal{U} \times M: \varphi(t, y, u) \in \mathcal{H}_{x} \forall t \in \mathbb{R}\right\}
$$

O conjunto $\mathbb{D}_{x}$ também é invariante pelo fluxo de controle $\Phi$ mas pode conter pontos que não são necessariamente pontos periódicos de $\Phi$. No entanto, temos a seguinte relação entre $\mathbb{D}_{x}$ e $\mathrm{H}_{x}$ :

Lema 3.2 Para todo $x \in \mathcal{P}$ temos $\overline{\mathrm{H}_{x}}=\overline{\mathbb{D}_{x}}$.

Demonstração. Primeiramente vamos mostrar que $\mathrm{H}_{x} \subset \mathbb{D}_{x}$.

Seja $(v, y) \in \mathbf{H}_{x}$. Pela definição de $\mathbf{H}_{x},(v, y) \in H_{(u, x)}$ para algum controle $u \in \mathcal{U}_{x}, \operatorname{logo} \varphi(t, y, v) \in \mathcal{H}_{x} \forall t \in \mathbb{R}$, isto é, $(v, y) \in \mathbb{D}_{x}$.

Mostraremos agora que $\mathbb{D}_{x} \subset \overline{\mathbf{H}}_{x}$. Dado $(v, y) \in \mathbb{D}_{x}$ vamos mostrar que para qualquer $\varepsilon>0, \exists\left(v_{p}, y\right) \in \mathbf{H}_{x} \operatorname{com} d\left((v, y),\left(v_{p}, y\right)\right)<\varepsilon$. Para tanto, vamos construir um controle periódico $v_{p} \in \mathcal{U}$ tal que $\left(v_{p}, y\right)$ seja um ponto periódico de $\Phi$ e $d_{\mathcal{U}}\left(v, v_{p}\right)<\varepsilon$.

Seja $N \in \mathbb{N}$ tal que

$$
\sum_{n=N+1}^{\infty} 2^{-n}<\frac{\varepsilon}{2}
$$

e $T>0$ tal que

$$
\int_{\mathbb{R} \backslash[-T, T]}\left|x_{i}(t)\right| d t<\frac{\varepsilon}{2 \operatorname{diâm} U}
$$

para $i \in\{1, \ldots, N\}$, onde $\left(x_{i}\right)_{i}$ é a sequência densa em $L^{1}\left(\mathbb{R}, \mathbb{R}^{m}\right)$ que foi usada no lema 1.3 para definir a distância $d_{\mathcal{U}}$.

Como $(v, y) \in \mathbb{D}_{x}, \varphi(-T, y, v)$ e $\varphi(T, y, v) \in \mathcal{H}_{x}$, então, pela controlabilidade em tempo finito em $\mathcal{H}_{x}$, existe $T_{1}>0$ e $u^{0} \in \mathcal{U}$ com

$$
\varphi\left(T_{1}, \varphi(T, y, v), u_{0}\right)=\varphi(-T, y, v) .
$$

Definimos

$$
v_{p}(t)=\left\{\begin{array}{ll}
v(t) & \text { se } t \in[-T, T] \\
u^{0}(t-T) & \text { se } t \in\left(T, T+T_{1}\right)
\end{array},\right.
$$

e estendemos $v_{p}$ como uma função $\left(2 T+T_{1}\right)$-periódica em $\mathbb{R}$. A trajetória correspondente $\varphi\left(\cdot, y, v_{p}\right)$ é também periódica com período $2 T+T_{1}$ e, portanto, 
está contida em $\mathcal{H}_{x}$ pela maximalidade dos conjuntos de controlabilidade total. Então $\left(v_{p}, y\right)$ é um ponto $\Phi$-periódico $\mathrm{em}_{x}$. Além disso,

$$
\left|\int_{\mathbb{R}}\left\langle v(t)-v_{p}(t), x_{i}(t)\right\rangle d t\right| \leq \operatorname{diâm} U \cdot \int_{\mathbb{R} \backslash[-T, T]}\left|x_{i}(t)\right| d t<\frac{\varepsilon}{2}
$$

para qualquer $i \in\{1, \ldots, N\}$. Agora,

$$
\begin{aligned}
d_{\mathcal{U}}\left(v, v_{p}\right) & <\sum_{n=1}^{N} \frac{1}{2^{n}} \frac{\left|\int_{\mathbb{R}}\left\langle v(t)-v_{p}(t), x_{n}(t)\right\rangle d t\right|}{1+\left|\int_{\mathbb{R}}\left\langle v(t)-v_{p}(t), x_{n}(t)\right\rangle d t\right|}+\frac{\varepsilon}{2} \\
& <\sum_{n=1}^{N} \frac{1}{2^{n}} \frac{\varepsilon}{2}+\frac{\varepsilon}{2}<\varepsilon .
\end{aligned}
$$

Dada a generalidade na escolha de $\varepsilon$ temos $(v, y) \in \overline{\mathbf{H}_{x}}$. Logo

$$
\mathrm{H}_{x} \subset \mathbb{D}_{x} \subset \overline{\mathrm{H}_{x}}
$$

o que demonstra o lema.

Definição 3.2 Um conjunto $X \in \mathcal{U} \times M$ é chamado de topologicamente transitivo em relação ao fluxo $\Phi$, se existe algum $z \in X$ tal que

$$
\omega(z):=\bigcap_{s>0} \overline{\Phi([s, \infty), z)}=X
$$

e é chamado topologicamente mixing em relação a $\Phi$ se, para quaisquer dois conjuntos abertos $V_{1}$ e $V_{2}$ de $X$, existem $T_{0} \in \mathbb{R}$ e $T_{1}>0$ tais que, para todo $n \in \mathbb{N}$, tenhamos

$$
\Phi\left(-n T_{1}+T_{0}, V_{1}\right) \cap V_{2} \neq \emptyset .
$$

O lema 3.2 nos permite generalizar a proposição 3.5, pág. 477 de [10], para sistemas de controle que não são necessariamente localmente acessíveis. Além disso, permite evitar a hipótese que o interior dos conjuntos controláveis deve ser não vazio. O teorema a seguir mostra a relação entre os conjuntos topologicamente mixing e topologicamente transitivos de $\Phi$ com o fecho $\overline{\mathrm{H}_{x}}$ dos levantamentos dos conjuntos de controlabilidade total. Além disso, o ítem 3 mostra que o sistema dinâmico $\Phi$ restrito ao conjunto $\overline{\mathrm{H}_{x}}$ é caótico no sentido de Devaney [18]. Veja também a seção 3.3 para uma comparação mais precisa entre o resultado de [10] e os resultados do teorema 3.3 desta tese. 
Teorema 3.3 Para qualquer $x \in \mathcal{P}$, temos que:

1. $\overline{\mathrm{H}_{x}}$ é um conjunto topologicamente mixing em relação a $\Phi$.

2. Se $\mathcal{H}_{x}$ é limitado em $M$, então $\overline{\mathrm{H}_{x}}$ é um conjunto topologicamente transitivo em relação a $\Phi$.

3. Se $\mathcal{H}_{x}$ é limitado em $M$, então a aplicação $\Phi$ possui dependência sensitiva nas condições iniciais em $\overline{\mathrm{H}_{x}}$.

Demonstração. Para mostrar a primeira afirmação, vamos mostrar que para quaisquer pares $W_{1}, W_{2}$ de conjuntos abertos de $\overline{\mathbf{H}_{x}}$, existe $T_{0} \in \mathbb{R}$ e $T_{1}>0$ tal que para todo todo $n \in \mathbb{N}, \Phi\left(-n T_{1}+T_{0}, W_{2}\right) \cap W_{1} \neq \emptyset$.

Sejam $\left(u_{i}, x_{i}\right) \in W_{i} \cap \mathbf{H}_{x}$ e $\varphi\left(\cdot, x_{i}, u_{i}\right)$ trajetórias $t_{i}$-periódicas contidas em $\mathcal{H}_{x}$ para respectivos $t_{i}>0, i=1,2$. É suficiente considerar $W_{i}$ da forma

$$
W_{i}=\left(V_{i} \times N_{i}\right) \cap \overline{\mathbf{H}_{x}},
$$

onde

$N_{i}=\left\{y \in M: d\left(x_{i}, y\right)<\varepsilon\right\}$ e $V_{i}=\left\{v \in \mathcal{U}:\left|\int_{\mathbb{R}}\left\langle u_{i}(\tau)-v(\tau), y_{i j}(\tau)\right\rangle d \tau\right|<\varepsilon\right\}$,

para $i=1,2$.

Existe $T>0$ tal que, para $i=1,2, j=1, \ldots, k_{i}$

$$
\int_{\mathbb{R} \backslash[-T, T]}\left|y_{i j}(t)\right| d t<\frac{\varepsilon}{\operatorname{diâm} U} .
$$

Podemos assumir que $t_{1}, t_{2}>T$. Devido a controlabilidade exata em $\mathcal{H}_{x}$, existem $t_{0}>0$ e $v_{0} \in \mathcal{U} \operatorname{com} \varphi\left(t_{0}, x_{2}, v_{0}\right)=x_{1}$. Tomamos $n \in \mathbb{N}$ fixo. Definimos $v_{n} \in \mathcal{U}$ por

$$
v_{n}(\tau)= \begin{cases}u_{1}(\tau) & \tau \in\left[-n t_{1}, \infty\right) \\ v_{0}\left(\tau+n t_{1}+t_{0}\right) & \tau \in\left[-n t_{1}-t_{0},-n t_{1}\right) \\ u_{2}\left(\tau+n t_{1}+t_{0}\right) & \tau \in\left(-\infty,-n t_{1}-t_{0}\right]\end{cases}
$$

Então $v_{n} \in V_{1}$, pois

$$
\left|\int_{\mathbb{R}}\left\langle u_{1}(\tau)-v_{n}(\tau), y_{1 j}(\tau)\right\rangle d \tau\right| \leq \operatorname{diâm} U \cdot \int_{\mathbb{R} \backslash\left[-t_{1}, t_{1}\right]}\left|y_{1 j}(\tau)\right| d \tau<\varepsilon .
$$


Além disso

$$
\begin{aligned}
& \varphi\left(t, x_{1}, v_{n}\right)=\varphi\left(t, x_{1}, u_{1}\right), \text { para } t \geq-n t_{1} \mathrm{e} \\
& \varphi\left(t, x_{1}, v_{n}\right)=\varphi\left(t+n t_{1}+t_{0}, x_{2}, u_{2}\right), \text { para } t<-n t_{1}-t_{0} .
\end{aligned}
$$

Assim, $\varphi\left(t, x_{1}, v_{n}\right) \in \mathcal{H}_{x}$ para $t \in \mathbb{R}$ e, portanto, $\left(v_{n}, x_{1}\right) \in \mathbb{D}_{x}$. Pelo lema (3.2), $\left(v_{n}, x_{1}\right) \in \overline{\mathbf{H}_{x}}$. Logo $\left(v_{n}, x_{1}\right) \in W_{1}$. Por outro lado, observe que

$$
\begin{aligned}
\varphi\left(-n t_{1}-t_{0}-t_{2}, x_{1}, v_{n}\right) & =x_{2} \mathrm{e} \\
v_{n}\left(-n t_{1}-t_{0}-t_{2}+\tau\right) & =u_{2}\left(\tau-t_{2}\right)=u_{2}(\tau) \text { para } \tau<t_{2} .
\end{aligned}
$$

Então, para todo $j=1, \ldots, k_{2}$, temos

$$
\begin{gathered}
\left|\int_{\mathbb{R}}\left\langle u_{2}(\tau)-v_{n}\left(-n t_{1}-t_{0}-t_{2}+\tau\right), y_{2 j}(\tau)\right\rangle d \tau\right|= \\
\left|\int_{t_{2}}^{\infty}\left\langle u_{2}(\tau)-v_{n}\left(-n t_{1}-t_{0}-t_{2}+\tau\right), y_{2 j}(\tau)\right\rangle d \tau\right| \leq \\
\operatorname{diâm} U \cdot \int_{t_{2}}^{\infty}\left|y_{2 j}(\tau)\right| d \tau<\varepsilon .
\end{gathered}
$$

Então

$$
\begin{gathered}
\Phi\left(-n t_{1}-t_{0}-t_{2},\left(v_{n}, x_{1}\right)\right)= \\
\left(v_{n}\left(-n t_{1}-t_{0}-t_{2}+\cdot\right), \varphi\left(-n t_{1}-t_{0}-t_{2}, x_{1}, v_{n}\right)\right)= \\
\left(\theta\left(-n t_{1}-\left(t_{0}+t_{2}\right), v_{n}\right), x_{2}\right) \in W_{2} .
\end{gathered}
$$

O ítem 2 decorre do fato que se um conjunto compacto é topologicamente mixing então é também topologicamente transitivo ([30], pág. 88).

O ítem 3 decorre do fato que, pela sua própria definição, o conjunto $\overline{\mathbf{H}_{x}}$ possui um conjunto denso de pontos periódicos e, pelo ítem $1, \overline{\mathbf{H}_{x}}$ é topologicamente mixing. Em [4], está demonstrado que estas duas propriedades juntas implicam dependência sensitiva em relação as condições iniciais, isto é, existe $\delta>0$ tal que, para todo $(u, y) \in \overline{\mathbf{H}_{x}}$ e para cada vizinhança $W$ de $(u, y)$ em $\overline{\mathbf{H}_{x}}$, existe $(v, z) \in W$ e $t>0$ de maneira que $\tilde{d}\left(\Phi^{t}(u, y), \Phi^{t}(\underset{\sim}{v}, z)\right)>\delta$, onde $\tilde{d}$ é a distância em $\mathcal{U} \times M$ definida como, por exemplo, $\tilde{d}((u, y),(v, z))=d_{\mathcal{U}}(u, v)+d_{M}(y, z)$, onde $d_{\mathcal{U}}$ é a distância definida em (1.5) e $d_{M}$ é uma distância em $M$.

Vale observar que nem todos os conjuntos topologicamente mixing do sistema dinâmico $\Phi$ estão relacionados com o conjunto $\mathcal{P}$. Como um exemplo, considere o fluxo irracional no toro (pensado como um sistema de controle), conforme exemplo 2.2. Neste caso, todo conjunto $\mathcal{U} \times M=\{0\} \times T^{2}$ é topologicamente mixing, no entanto, $\mathcal{H}_{x}=\emptyset$ para todo $x \in T^{2}$. 


\subsection{Notas.}

Na seção 2.3, há uma conparação entre os conjuntos controláveis $D$ e outros conjuntos recorrentes como $\mathcal{D}_{x}^{\sharp}$ e os conjuntos de controlabilidade aproximada $\mathcal{D}_{x}$. O exemplo 2.3 mostra que, em geral, não podemos considerar o levantamento dos conjuntos $\mathcal{D}_{x}^{\sharp}$ e $\mathcal{D}_{x}$ pois, para alguns pontos $y$ destes conjuntos, nem sempre existe $u \in \mathcal{U}$ tal que $\varphi(t, y, u)$ permaneça no conjunto para todo $t>0$. Em [10] está estabelecida uma conexão entre os conjuntos topologicamente mixing de $\Phi$ e o levantamento ao produto $\mathcal{U} \times M$ dos Conjuntos Controláveis, isto é, foi considerado o fecho do conjunto

$$
\mathrm{D}=\{(u, x) \in \mathcal{U} \times M: \varphi(t, x, u) \in \operatorname{int} D \forall t \in \mathbb{R}\},
$$

onde $D$ é um conjunto controlável de interior não vazio. Lá são considerados apenas os sistemas de controle localmente acessíveis. Neste caso, conforme a proposição 2.17 e o corolário 2.18 da presente tese, temos $\overline{\mathrm{D}}=\overline{\mathrm{H}_{x}}$. No entanto, sob estas hipóteses, o resultado obtido em [10] é mais específico, no sentido que estabelece uma correspondência biunívoca entre os conjuntos topologicamente mixing de $\Phi$ que possuem uma projeção em $M$ com interior não vazio e o levantamento dos conjuntos controláveis de interior não vazio.

Note que, no exemplo citado na observação feita logo após o teorema 3.3, o sistema não é localmente acessível.

$\mathrm{Na}$ verdade, a tentativa de generalizar os resultados de [10] foi o que norteou a construção da presente tese. 


\section{Capítulo 4}

\section{Perturbação de sistemas lineares.}

Neste capítulo, estudaremos os conjuntos de controlabilidade total para o caso específico dos sistemas lineares e para as perturbações dos sistemas lineares de controle. Veremos que a estrutura do conjunto $\mathcal{P}$ dos pontos periódicos de um sistema de controle linear permanece inalterada para perturbações suficientemente pequenas de um sistema de controle linear hiperbólico.

Os resultados deste capítulo foram adaptados de [16] e [17], onde foi estudada a unicidade dos conjuntos controláveis para perturbações de sistemas lineares hiperbólicos.

\subsection{Sistemas Lineares}

Nesta subseção vamos analisar em mais detalhes o que acontece com sistemas de controle lineares. Mais especificamente, vamos considerar sistemas do tipo

$$
\dot{x}=A x+B u
$$

onde $x \in R^{n}$, $A$ é uma matriz $n \times n$ e $\mathrm{B}$ é uma matriz $n \times m$, ambas com coeficientes reais. $\mathrm{O}$ conjunto de controles admissíveis é $\mathcal{U}=\{u: \mathbb{R} \rightarrow$ $\mathbb{R}^{m}$ mensurável: $u(t) \subset U$ quase sempre $\}$, onde $U \subset \mathbb{R}^{m}$ é um conjunto compacto e convexo.

Definição 4.1 Diz-se que uma matriz A é hiperbólica se A não possui autovalores com parte real nula. Um sistema de controle do tipo 4.1 com a matriz A hiperbólica é chamado de sistema linear hiperbólico. 
Analisaremos primeiramente as questões de existência de trajetórias periódicas do sistema (4.1) com a matriz $A$ hiperbólica. O seguinte lema, que está publicado em [17], terá um papel importante no que segue.

Lema 4.1 Se a matriz $A$ é hiperbólica, então existe uma constante $K_{A}>0$, que depende apenas de $A$ tal que, para todo $T>0$ e $y \in L_{T}^{\infty}\left(\mathbb{R}, \mathbb{R}^{n}\right)=$ $\left\{y \in L^{\infty}\left(\mathbb{R}, \mathbb{R}^{n}\right): y(t+T)=y(t) \forall t \in \mathbb{R}\right\}$, existe uma única solução T-periódica $\xi$ de

$$
\dot{x}=A x+y
$$

e esta solução satisfaz

$$
\|\xi\|_{W^{1, \infty}}<K_{A}\|y\|_{L^{\infty}}
$$

onde $\|y\|_{L^{\infty}}=\operatorname{supess}_{t \in \mathbb{R}}\|y(t)\| e\|\xi\|_{W^{1, \infty}}=\max \left\{\|y\|_{L^{\infty}},\|\dot{y}\|_{L^{\infty}}\right\}$.

Demonstração. Como $A$ é hiperbólica, para qualquer função y $T$ periódica, a solução de 4.2 é dada por

$$
\xi(t)=e^{t A}\left(I-e^{T A}\right)^{-1} \int_{0}^{T} e^{(T-s) A} y(s) d s+\int_{0}^{t} e^{(t-s) A} y(s) d s
$$

Para mostrar (4.3), primeiro mostraremos que para toda função (não necessariamente periódica) $y \in L^{\infty}\left(\mathbb{R}, \mathbb{R}^{n}\right)$ existe uma solução essencialmente limitada de (4.2).

Considere $P$ a projeção de $\mathbb{R}^{n}$ sobre o espaço vetorial $E_{\text {est }}$, que é a soma direta dos auto-espaços generalizados associados aos auto-valores com parte real negativa. Temos que

$$
\begin{aligned}
\left\|e^{t A}(I-P) e^{-s A}\right\| & \leq K e^{-a(t-s)} \text { para } t \geq s, \\
\left\|e^{t A} P e^{-s A}\right\| & \leq L e^{-b(t-s)} \text { para } s \geq t
\end{aligned}
$$

onde $K, L, a, b$ são constantes positivas. Portanto, a solução

$$
\xi(t)=\int_{-\infty}^{t} e^{t A}(I-P) e^{-s A} y(s) d s-\int_{t}^{\infty} e^{t A} P e^{-s A} y(s) d s
$$

é essencialmente limitada.

Considere a aplicação linear $\Psi: W^{1, \infty}\left(\mathbb{R}, \mathbb{R}^{n}\right) \rightarrow L^{\infty}\left(\mathbb{R}, \mathbb{R}^{n}\right)$ que leva $x \mathrm{em}$ $\dot{x}-A x$. Como $x \equiv 0$ é a única solução essencialmente limitada de $\Psi(x)=0$, 
a aplicação $\Psi$ é injetiva. $\Psi$ é obviamente contínua e 4.4 mostra que $\Psi$ é também sobrejetiva. Então, existe uma constante $K_{A}$ tal que $\left\|\Psi^{-1}\right\| \leq K_{A}$, isto é, para qualquer $y$ essencialmente limitada, a solução $\xi$ de $\dot{x}=A x+y$ satisfaz

$$
\|\xi\|_{W^{1, \infty}}<K_{A}\|y\|_{L^{\infty}}
$$

o que demonstra o lema.

Lema 4.2 Em um sistema do tipo (4.1), com $0 \in U$, se o conjunto das trajetórias periódicas $\mathcal{P}$ é limitado, então A é hiperbólica.

Demonstração. Suponhamos que $A$ não seja hiperbólica. Se o determinante da matriz $A$ é igual a 0 temos que o subespaço $V=\left\{x \in \mathbb{R}^{n}: A x=0\right\}$ é diferente de $\{0\}$ e portanto ilimitado. Qualquer ponto de $V$ pode ser considerado uma trajetória periódica (na verdade um ponto fixo) quando usamos o controle $u(t) \equiv 0$. Portanto, qualquer ponto de $V$ está contido em $\mathcal{P}$. Se o determinante de $A$ for diferente de 0 , existe um par de auto valores imaginários conjugados $\pm i \beta \operatorname{com} \beta \neq 0$. A representação de $A$ na forma canônica real de Jordan, nos mostra que existe um subespaço $W$ de dimensão dois tal que

$$
A_{\mid W}=\left(\begin{array}{cc}
0 & \beta \\
-\beta & 0
\end{array}\right) \text {. }
$$

Usando-se novamente o controle $u(t) \equiv 0$, temos que qualquer ponto de $W$ é o começo de uma trajetória periódica de período $\frac{2 \pi}{\beta}$ e portanto pertence a $\mathcal{P}$.

O teorema abaixo caracteriza os sistemas lineares que possuem conjuntos de controlabilidade total limitados.

Teorema 4.3 Considere $U \subset \mathbb{R}^{m}$ tal que $0 \in U$. Então, o conjunto das trajetórias periódicas $\mathcal{P}$ do sistema 4.1 é limitado, se e somente se, a matriz A é hiperbólica.

Demonstração. Se o conjunto $\mathcal{P}$ é limitado, $A$ é hiperbólica pelo lema 4.2. Reciprocamente, se $A$ é hiperbólica e o conjunto $\mathcal{P}$ não é limitado, existe uma sequência ilimitada de trajetórias periódicas do sistema (4.1), o que contradiz a desigualdade (4.5), já que o $\sup _{u \in \mathcal{U}}\|u\|_{L^{\infty}} \leq \max \{|v|: v \in U\}$ é finito.

Para sistemas do tipo (4.1) com $0 \in U$, temos que $\mathcal{P} \neq \emptyset$, (pois $0 \in \mathcal{P}$ ). $\mathrm{O}$ conjunto $\mathcal{P}$ também é conexo, pois, dada a linearidade do sistema, se o 
controle $u$ gera uma trajetória periódica $\varphi$, para cada $\lambda \in[0,1]$, o controle $\lambda u$ (que pertence a $\mathcal{U}$ já que $U$ é convexo) irá gerar a trajetória periódica $\lambda \varphi$. Fazendo-se $\lambda$ variar entre 0 e 1 , podemos concluir que $\mathcal{P}$ é conexo.

No entanto, a estrutura mais fina em $\mathcal{P}$, representada pelos conjuntos de controlabilidade total, que particionam $\mathcal{P}$, pode ser bem diversa, mesmo em sistemas lineares com a matriz $A$ hiperbólica. No exemplo 1.1 temos 3 conjuntos de controlabilidade total, sendo um aberto e dois fechados. O exemplo 4.1 a seguir, mostra que podem existir infinitos conjuntos de controlabilidade total.

\section{Exemplo 4.1 No sistema}

$$
\left\{\begin{array}{l}
\dot{x}_{1}=x_{1}+u_{1} \\
\dot{x}_{2}=x_{2}+u_{2}
\end{array}\right.
$$

com $u(t)=\left(u_{1}(t), u_{2}(t)\right) \in U:=\left\{\left(u_{1}, u_{2}\right) \in \mathbb{R}^{2}: u_{1}^{2}+u_{2}^{2} \leq 1\right\}$ quase sempre, temos $\mathcal{H}_{0}=\left\{\left(x_{1}, x_{2}\right) \in \mathbb{R}^{2}: x_{1}^{2}+x_{2}^{2}<1\right\}$ e, se $p \in\left\{\left(x_{1}, x_{2}\right) \in \mathbb{R}^{2}: x_{1}^{2}+x_{2}^{2}=1\right\}$, vale que $\mathcal{H}_{p}=\{p\}$, isto é, cada ponto da circunferência de raio unitário $e$ centro na origem, representa um conjunto de controlabilidade total. Neste exemplo temos infinitos conjuntos de controlabilidade total.

Se restringirmos o conjunto de controles admissíveis a

$$
\text { int } \mathcal{U}:=\left\{u: \mathbb{R} \rightarrow \mathbb{R}^{m} \text { mensurável }: u(t) \in \operatorname{int} U \text { quase sempre }\right\} \text {. }
$$

nos sistemas onde int $U \neq \emptyset$, podemos provar que existe um único conjunto de controlabilidade total nos sistemas de controle lineares hiperbólicos controláveis (definição 4.2).

Definição 4.2 O par de matrizes $(A, B)$ da equação (4.1) é chamado de par controlável se

$$
\text { posto }\left[B, A B, \ldots, A^{n-1} B\right]=n \text {. }
$$

Neste caso, o sistema (4.1) é chamado de controlável.

O lema abaixo mostra que os conjuntos de acessibilidade e controlabilidade para sistemas de controle lineares controláveis, com int $\mathcal{U}$ como conjunto de controles admissíveis, são abertos. 
Lema 4.4 No sistema (4.1), se o par $(A, B)$ é controlável, então os conjuntos $\mathcal{O}^{+}(x)$ e $\mathcal{O}^{-}(x)$, definidos em (1.3), mas usando-se como controles admissíveis int $\mathcal{U}$, são-abertos.

Demonstração. Seja $y \in \mathcal{O}^{+}(x)$. Tome $t_{0} \in \mathbb{R}^{+}$e $u_{0} \in$ int $\mathcal{U}$ tal que $\varphi\left(t_{0}, x, u_{0}\right)=y$, onde $\varphi$ é a solução da equação (4.1) que começa em $x$ e usa o controle $u_{0}$. Considere o conjunto

$$
E_{t_{0}}=\left\{v \in C\left(\left[0, t_{0}\right] \rightarrow \mathbb{R}^{m}\right)\right\}
$$

e o conjunto

$$
\mathcal{V}_{\left(t_{0}, u_{0}\right)}=\left\{v \in E_{t_{0}}: u_{0}(t)+v(t) \in \operatorname{int} U \text { para } t \in\left[0, t_{0}\right]\right\} .
$$

$\mathcal{V}_{\left(t_{0}, u_{0}\right)}$ é um subconjunto aberto do espaço de Banach $E_{t_{0}}$.

Definimos

$$
\Theta: \mathcal{V}_{\left(t_{0}, u_{0}\right)} \rightarrow \mathbb{R}^{n}
$$

como

$$
\Theta(v)=\varphi\left(t_{0}, x, u_{0}+v\right)=e^{t_{0} A} x+\int_{0}^{t_{0}} e^{\left(t_{0}-s\right) A} B\left(u_{0}(s)+v(s)\right) d s .
$$

Note que $\Theta(0)=y$. Para $\omega \in E_{t_{0}}$ temos

$$
\Theta^{\prime}(0) \omega=\int_{0}^{t_{0}} e^{\left(t_{0}-s\right) A} B \omega(s) d s
$$

e a hipótese de controlabilidade implica que $\Theta^{\prime}(0)$ é sobrejetiva. Portanto, existe uma vizinhança $W$ de 0 em $\mathcal{V}_{\left(t_{0}, u_{0}\right)}$ tal que $y \in \operatorname{int}(\Theta(W)) \subset \mathcal{O}^{+}(x)$. Para mostrar que $\mathcal{O}^{-}(x)$ é aberto, o raciocínio é análogo, apenas trocando-se $t_{0}$ por $-t_{0}$.

Teorema 4.5 Se A é hiperbólica e o conjunto de controles admissíveis for intU, então existe um único conjunto de controlabilidade total para o sistema (4.1). Este conjunto é limitado, conexo, convexo e aberto referente a topologia do subespaço $V \subset \mathbb{R}^{n}$ gerado pelas colunas da matriz $\left[B, A B, \ldots, A^{n-1} B\right]$.

Demonstração. Suponhamos primeiramente que o posto da matriz $\left[B, A B, \ldots, A^{n-1} B\right]$ é igual a $n$. Pelo lema 4.4 , todos os conjuntos de controlabilidade total, por serem intersecções de conjuntos abertos, são eles próprios 
conjuntos abertos. Escolhendo-se $u \equiv 0$, resulta que a solução $\varphi(t, 0,0)$ do sistema 4.1 é identicamente nula e portanto, $\mathcal{H}(0)$ é um conjunto aberto não vazio.

Para demonstrar a unicidade, suponhamos que exista outro conjunto de controlabilidade total $\mathcal{H}_{1}$ e tome $x \in \mathcal{H}_{1}$. Como $\mathcal{H}_{1}$ é aberto, $\exists \varepsilon>0$ tal que $\mu x \in \mathcal{H}_{1} \forall \mu \in(1-\varepsilon, 1+\varepsilon)$. Fixado $\mu \in(1-\varepsilon, 1+\varepsilon)$, podemos escolher controles $u_{1}$ e $u_{2}$ tais que $\varphi\left(T_{1}, x, u_{1}\right)=\mu x$ e $\varphi\left(T_{2}, x, u_{2}\right)=x$ para certos $T_{1}, T_{2}>0$. Usando a linearidade do sistema e a convexidade de $U$, temos que, $\forall \alpha \in(0,1)$ vale

$$
\varphi\left(T_{1}, \alpha x, \alpha u_{1}\right)=\alpha \mu x \text { e } \varphi\left(T_{2}, \alpha x, \alpha u_{2}\right)=\alpha x
$$

o que implica $\alpha x \in \mathcal{H}_{1} \forall \alpha \in[0,1]$. Como $\mathcal{H}(0)$ é aberto, podemos concluir que $\mathcal{H}(0)=\mathcal{H}_{1}$, isto é, existe um único conjunto de controlabilidade total. Quanto a conexidade, basta notar que $\alpha x \in \mathcal{H}_{1} \forall \alpha \in[0,1]$.

Agora, se o posto de $\left[B, A B, \ldots, A^{n-1} B\right]=d<n$, podemos escolher uma base de $\mathbb{R}^{n}$ de tal forma que a equação (4.1) seja escrita como

$$
\left(\begin{array}{c}
\dot{x}_{1} \\
\dot{x}_{2}
\end{array}\right)=\left(\begin{array}{cc}
A_{11} & A_{12} \\
0 & A_{22}
\end{array}\right)\left(\begin{array}{l}
x_{1} \\
x_{2}
\end{array}\right)+\left(\begin{array}{c}
B_{1} \\
0
\end{array}\right) u
$$

onde $x_{1} \in \mathbb{R}^{d}$ e $x_{2} \in \mathbb{R}^{n-d}$ [23]. Note que a coordenada $x_{2}$ não depende dos controles. Como a matriz $A$ é hiperbólica, a matriz $A_{22}$ também é hiperbólica, o que implica que as órbitas periódicas do sistema (4.1), nesta nova base, possuem a coordenada $x_{2}$ identicamente nula. Por outro lado, o sistema restrito ao subespaço $\mathbb{R}^{d} \times 0$ é um sistema controlável e, portanto, $\mathcal{O}^{+}(0) \cap$ $\mathcal{O}^{-}(0)$ é um subconjunto aberto de $\mathbb{R}^{d}$ o que demonstra a última afirmação do teorema. Quanto a convexidade, é um fato geral para sistemas lineares que tanto $\mathcal{O}^{+}(x)$ como $\mathcal{O}^{-}(x)$ são conjuntos convexos, assim, $\mathcal{O}^{+}(x) \cap \mathcal{O}^{-}(x)$ também é convexo [28].

\subsection{Perturbação de sistemas lineares}

Nesta seção, vamos mostrar que quando o sistema de controle é uma perturbação (não necessariamente linear) de um sistema linear hiperbólico, os conjuntos de controlabilidade total são todos limitados e a união de todos eles forma um conjunto limitado e conexo. Isto é, para perturbações de sistemas lineares hiperbólicos, a estrutura do conjunto $\mathcal{P}$ permanece inalterada se comparada com o que acontece para sistemas lineares. 
Capítulo 4. Perturbação de sistemas lineares.

Consideraremos, nessa seção, sistemas do tipo

$$
\dot{x}(t)=A x(t)+F(x, u)
$$

onde $u(t) \in U$ quase sempre, $U \subset \mathbb{R}^{m}$ limitado, $A$ é uma matriz hiperbólica e $F: \mathbb{R}^{n} \times V \rightarrow \mathbb{R}^{n}$ é uma função de classe $C^{1} \operatorname{com} F(0,0)=0$ e $\left\|D_{1} F(x, u)\right\|<$ $M$ uniformemente. O conjunto $V$ é um aberto de $\mathbb{R}^{m}$ tal que $U \subset V$ e $M$ é uma constante positiva.

O lema abaixo, de certa forma, generaliza o lema 4.1.

Lema 4.6 [17] Para o sistema (4.8), existe uma constante $K_{A}$, dependendo somente de A tal que, se

$$
\left\|D_{1} F(x, u)\right\| \leq \min \left\{1, \frac{1}{2 K_{A}}\right\} \text {, uniformemente }
$$

então, para quaisquer $T>0$ e $u \in \mathcal{U}_{T}$, a equação (4.8) possui uma única solução T-periódica $x(\cdot, u)$ e a aplicação $\mathcal{U}_{T} \rightarrow W_{T}^{1, \infty}\left(\mathbb{R}^{n}\right)$ dada por $u \rightarrow$ $x(\cdot, u)$ é contínua. Se, além disso, $0 \in$ int $U$ temos que, para qualquer $u \in \mathcal{U}_{T}$,

$$
\sup _{t \in[0, t]}|x(t, u)| \leq 2 R_{U} K_{A}\left(\operatorname{supess}_{u \in U}\|F(0, u)\|\right) \text {, }
$$

onde $R_{U}:=\max \{|v|: v \in U\}$.

Demonstração. Vamos reescrever a equação (4.8) da seguinte forma:

$$
L x-\bar{A} x-\bar{F}(x, u)=0
$$

onde

$$
\begin{array}{lll}
L: W_{T}^{1, \infty}\left(\mathbb{R}^{d}\right) \rightarrow L_{T}^{\infty}\left(\mathbb{R}^{d}\right) & \operatorname{com} & (L x)(t)=\dot{x}(t), \\
\bar{A}: W_{T}^{1, \infty}\left(\mathbb{R}^{d}\right) \rightarrow L_{T}^{\infty}\left(\mathbb{R}^{d}\right) & \operatorname{com} & (A x)(t)=A x(t), \\
\bar{F}: \mathcal{U}_{T} \times W_{T}^{1, \infty}\left(\mathbb{R}^{d}\right) \rightarrow L_{T}^{\infty}\left(\mathbb{R}^{d}\right) & \operatorname{com} & \bar{F}(x, u)(t)=F(x(t), u(t)) .
\end{array}
$$

Pelo lema 4.1, $(L-\bar{A}) x=y$ implica $\|x\|_{W^{1, \infty}}<K_{A}\|y\|_{L^{\infty}}$ e então

$$
\left\|(L-\bar{A})^{-1}\right\| \leq K_{A} .
$$

Seja $\Phi: \mathcal{U}_{T} \times W_{T}^{1, \infty}\left(\mathbb{R}^{n}\right) \rightarrow W_{T}^{1, \infty}\left(\mathbb{R}^{n}\right)$, dada por

$$
\Phi(x, u)=-(L-\bar{A})^{-1}(\bar{F}(x, u)) .
$$


Então a equação (4.8) é equivalente a

$$
\Phi(x, u)=x .
$$

Vamos mostrar agora que a equação (4.8) possui exatamente uma solução para qualquer $u \in \mathcal{U}_{T}$. Como

$$
\begin{aligned}
\left\|\Phi\left(x_{1}, u\right)-\Phi\left(x_{2}, u\right)\right\|_{W^{1, \infty}} & \leq\left\|(L-\bar{A})^{-1}\right\|\left\|\bar{F}\left(x_{1}, u\right)-\bar{F}\left(x_{2}, u\right)\right\|_{L^{\infty}} \\
& \leq K_{A} \sup _{(p, v) \in \mathbb{R}^{n} \times U}\left\|D_{1} F(p, v)\right\|\left\|x_{1}-x_{2}\right\|_{W^{1, \infty}} \\
& \leq \frac{1}{2}\left\|x_{1}-x_{2}\right\|_{W^{1, \infty}},
\end{aligned}
$$

para qualquer $u \in \mathcal{U}_{T}$, a aplicação $\Phi(\cdot, u)$ é uma contração. Assim, pelo teorema da contração de Banach, decorre a existência de um único ponto fixo que denotamos por $x(\cdot, u)$. Além disso, para $T>0$ fixo, a solução $x(\cdot, u)$ depende continuamente de $u \in \mathcal{U}_{T}$.

Para mostrar a última afirmação, note que para um ponto fixo $x$ de $\Phi(u, \cdot)$ temos

$$
\begin{aligned}
\|x\|_{W^{1, \infty}} & =\|\Phi(x, u)-\Phi(0,0)\|_{W^{1, \infty}} \\
& \leq\|\Phi(x, u)-\Phi(0, u)\|_{W^{1, \infty}}+\|\Phi(0, u)-\Phi(0,0)\|_{W^{1, \infty}} \\
& \leq \frac{1}{2}\|x\|_{W^{1, \infty}}+\left\|(L-\bar{A})^{-1}\right\|\left(\|\bar{F}(0, u)-\bar{F}(0,0)\|_{L^{\infty}}\right) \\
& \leq \frac{1}{2}\|x\|_{W^{1, \infty}}+K_{A}\left(\underset{u \in U}{\left.\operatorname{supess}_{u \in}\|F(0, u)\|\right) R_{U},}\right.
\end{aligned}
$$

e isto implica (4.9).

O corolário abaixo mostra que o conjunto $\mathcal{P}$ dos sistemas do tipo (4.8) são limitados.

Corolário 4.7 Para o sistema de controle

$$
\dot{x}=A x+F(x, u)
$$

com a matriz $A$ hiperbólica e a função $F$ satisfazendo

$$
F(0,0)=0 \quad\left\|D_{1} F(x, u)\right\|<\min \left\{1, \frac{1}{2 K}\right\} \quad \text { e } \quad\left\|D_{2} F(x, u)\right\|<M,
$$

e $u(t) \in U$ um conjunto limitado com $0 \in$ int $U$, temos que qualquer conjunto de controlabilidade total $\mathcal{H}_{\alpha}$ está contido na bola de centro na origem e raio $2 R_{U} K_{A} M$. 
Demonstração. Basta notar que se $x \in \mathcal{H}_{\alpha}$ então $x$ pertence a alguma trajetória periódica e portanto deve satisfazer (4.9).

Se o conjunto $U$ for convexo, o conjunto $\mathcal{P}$ será um conjunto conexo, conforme teorema a seguir.

Teorema 4.8 Se F satisfaz as hipóteses do corolário 4.7 e U é um conjunto convexo e limitado, então $\mathcal{P}$ é um conjunto conexo e limitado (que contém a origem).

Demonstração. Que $\mathcal{P}$ é limitado é o resultado do corolário 4.7. Para a conexidade de $\mathcal{P}$, considere $T>1$ e $u_{0} \in \mathcal{U}_{T}$. Então, o lema 4.1 implica que existe pelo menos um conjunto de controlabilidade total não vazio. Vamos assumir que existem dois conjuntos de controlabilidade total $\mathcal{H}_{\alpha_{0}}$ e $\mathcal{H}_{\alpha_{1}}$ que estão contidos em componentes conexos diferentes de $\mathcal{P}=\bigcup_{\alpha \in \Gamma} \mathcal{H}_{\alpha}$.

Para cada $x_{i} \in \mathcal{H}_{\alpha_{i}}, \quad i \in\{0,1\}, \exists u_{i} \in \mathcal{U}_{T_{i}} \operatorname{com} \varphi\left(T_{i}, x_{i}, u_{i}\right)=x_{i} \mathrm{e}$ $\varphi\left(t, x_{i}, u_{i}\right) \in \mathcal{H}_{\alpha_{i}} \forall t \in \mathbb{R}$. Podemos assumir $T_{i}>1$ pois se $u \in \mathcal{U}_{T}$ então $u \in \mathcal{U}_{n T}$ para qualquer $n \in \mathbb{N}$. Definimos

$$
\begin{aligned}
T_{\lambda} & =\lambda T_{1}+(1-\lambda) T_{0} \mathrm{e} \\
u_{\lambda}(t) & =\lambda u_{1}\left(\frac{T_{1} t}{T_{\lambda}}\right)+(1-\lambda) u_{0}\left(\frac{T_{0} t}{T_{\lambda}}\right) .
\end{aligned}
$$

Como $U$ foi assumido convexo $u_{\lambda} \in \mathcal{U}_{T_{\lambda}} \forall \lambda \in[0,1]$. Pelo lema 4.1, a equação

$$
\dot{x}=A x+F\left(x, u_{\lambda}\right)
$$

admite uma única solução $T_{\lambda}$-periódica $x_{\lambda}$ contida em algum $\mathcal{H}_{\beta}, \beta \in \Gamma$. Vamos mostrar que estas imagems formam um contínuo ligando $\mathcal{H}_{\alpha_{0}}$ a $\mathcal{H}_{\alpha_{1}}$ contradizendo a hipótese de existirem dois conjuntos de controlabilidade total.

Por uma mudança de escala no tempo, podemos transformar cada solução $T_{\lambda}$-periódica em uma solução 1-periódica do sistema

$$
\dot{\xi}(\tau)=\left[A \xi(\tau)+F\left(\xi(\tau), u_{\lambda}\left(\tau T_{\lambda}\right)\right)\right] T_{\lambda}
$$

e observe que $\hat{x}(\lambda, \tau)=x_{\lambda}\left(\tau T_{\lambda}\right)$ nos dá sua única solução 1-periódica. Uma vez que a aplicação $(\lambda, \tau) \rightarrow \hat{x}(\lambda, \tau)$ é contínua, o conjunto $\hat{x}([0,1] \times[0,1])$ é conexo e coincide com a união das imagens das curvas $x_{\lambda}(\cdot)$, para $\lambda \in[0,1]$. Usando $u \equiv 0$, resulta que a origem é um conjunto de controlabilidade total. 
Como uma aplicação do lema (4.6), vamos estudar sistemas bilineares, isto é, sistemas do tipo

$$
\dot{x}=A x+\sum_{i=1}^{m} u_{i} B_{i} x,
$$

onde $u(t)=\left(u_{1}(t), \ldots, u_{m}(t)\right) \in \mathcal{U}$ e $B_{i}: \mathbb{R}^{n} \rightarrow \mathbb{R}^{n}$ são operadores lineares constantes para todo $i \in\{1, \ldots, m\}$. Neste caso, $\bar{F}(x, u)=\sum_{i=1}^{m} u_{i} B_{i} x$ o que implica que $\bar{F}(0, u) \equiv 0$. De acordo com o lema (4.6), podemos concluir que, se $A$ é hiperbólica e $\left\|D_{1} F(x, u)\right\|$ for suficientemente pequeno, a origem é o único conjunto de controlabilidade total para o sistema (4.10), ou em outras palavras, a solução constante igual a zero é a única solução periódica do sistema (4.10).

A forma particular deste sistema implica que o tamanho de $\left\|D_{1} F(x, u)\right\|$ é pequeno se o domínio de controle $U$ for pequeno. Para ver isso, vamos introduzir um parametro $\rho>0$ para regular o diâmetro de $U$, isto é, dado $\rho>0$ considere o conjunto

$$
\rho U:=\{\rho u: u \in U\}
$$

e o conjunto de controles admissíveis

$$
\mathcal{U}^{\rho}:=\left\{u: \mathbb{R} \rightarrow \mathbb{R}^{m} \text { mensurável : } u(t) \subset \rho U \text { quase sempre }\right\} .
$$

Observe que, para $v \in \mathbb{R}^{n}, D_{1} F(x, u) \cdot v=\sum_{i=1}^{m} u_{i} B_{i} v$ o que implica que $\left\|D_{1} F(x, u)\right\| \leq R_{U} \sum_{i=1}^{m}\left\|B_{i}\right\|$ onde $R_{U}=\max \{|u|: u \in U\}$. Desta forma, quando nos restringimos a usar controles do tipo (4.11) temos

$$
\left\|D_{1} F(x, u)\right\| \leq \rho R_{U} \sum_{i=1}^{m}\left\|B_{i}\right\|
$$

e assim, para $\rho$ suficientemente pequeno podemos garantir que o sistema (4.10), com a matriz $A$ hiperbólica, possui a origem como sua única órbita periódica.

Agora vamos estabelecer algumas condições para que $\mathcal{H}(0)$ não se reduza a um único ponto. Na verdade, sob certas hipóteses sobre $F$ podemos garantir que $\mathcal{H}(0)$ é um conjunto aberto não vazio. 
Expandindo a função $F$ em série de Taylor, o sistema (4.8) pode ser escrito como

$$
\dot{x}=A x+F(0,0)+D_{1} F(0,0) x+D_{2} F(0,0) u+\tilde{R}(x, u)
$$

ou ainda, já que $F(0,0)=0$,

$$
\dot{x}=A x+D_{2} F(0,0) u+R(x, u)
$$

onde $R(x, u)=D_{1} F(0,0) x+\tilde{R}(x, u)$.

Os lemas 4.9 e 4.10 a seguir, apresentam uma generalização do lema 4.4 e são adaptações da proposição 3.1 de [17]. Convém observar que, em ambos os lemas, a matriz $A$ não precisa ser hiperbólica.

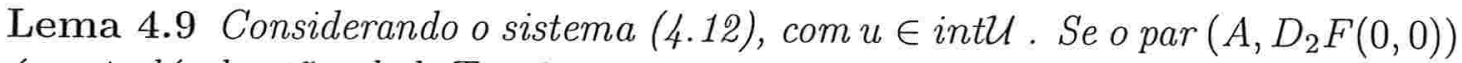
é controlável então, dado $T>0$ existe uma constante $M:=M\left(A, D_{2} F(0,0), T\right)$ tal que, para qualquer função $R$ de classe $C^{1}$, com $\left\|D_{1} R\right\|<M$ e $\left\|D_{2} R\right\|<$ $M$ uniformemente, temos: Para qualquer $x_{0} \in \mathbb{R}^{n}$ os conjuntos $\mathcal{O}_{T}^{+}\left(x_{0}\right)$ e $\mathcal{O}_{T}^{-}\left(x_{0}\right)$ são abertos.

Demonstração. Vamos seguir os passos da demonstração do Lema (4.4) e definir, para quaisquer $x_{0} \in \mathbb{R}^{n}, u_{0} \in$ intน e $T>0$, a aplicação

$$
\begin{gathered}
\Psi: \mathcal{V}_{\left(T, u_{0}\right)} \rightarrow \mathbb{R}^{n} \\
\Psi(v)=\varphi\left(T, x_{0}, u_{0}+v\right),
\end{gathered}
$$

onde $\varphi\left(t, x_{0}, u_{0}\right)$ é a solução de (4.12) com condição inicial $x_{0}$ usando controle $u_{0}$ e o conjunto $\mathcal{V}_{\left(T, u_{0}\right)}$ é um conjunto aberto do espaço de Banach $E_{T}$ definido de maneira análoga a (4.6). Vamos mostrar que $\Psi^{\prime}(0)=D_{3}\left(\varphi\left(T, x_{0}, u_{0}\right)\right)$ é sobrejetiva e concluir que $y=\varphi\left(T, x_{0}, u_{0}\right)$ está contido em uma vizinhança aberta de $\mathcal{O}_{T}^{+}\left(x_{0}\right)$.

Para $v \in C\left([0, T], \mathbb{R}^{n}\right)$, vamos definir

$$
\alpha(t)=D_{3} \varphi\left(t, x_{0}, u_{0}\right) \cdot v \quad \text { e } \beta(t)=D_{3} \psi\left(t, x_{0}, u_{0}\right) \cdot v
$$

onde $\psi\left(t, x_{0}, u_{0}\right)$ é a solução da equação

$$
\dot{x}=A x+D_{2} F(0,0) u, \quad x(0)=x_{0} .
$$

Usando a regra da cadeia temos,

$$
\dot{\alpha}(t)=\frac{d}{d t} D_{3} \varphi\left(t, x_{0}, u_{0}\right) \cdot v=
$$


Capítulo 4. Perturbação de sistemas lineares.

$$
\begin{aligned}
=D_{3} & \left(A \varphi\left(t, x_{0}, u_{0}\right)+D_{2} F(0,0) \cdot u_{0}+R\left(\varphi\left(t, x_{0}, u_{0}\right), u_{0}\right)\right) \cdot v= \\
= & A \alpha(t)+D_{2} F(0,0) \cdot v(t)+D_{1} R\left(\varphi\left(t, x_{0}, u_{0}\right), u_{0}\right) \cdot \alpha(t)+ \\
& +D_{2} R\left(\varphi\left(t, x_{0}, u_{0}\right), u_{0}\right) \cdot v(t) .
\end{aligned}
$$

Portanto

$$
\begin{gathered}
\alpha(t)= \\
=\int_{0}^{t} A \alpha(s)+D_{2} F(0,0) v(s)+D_{1} R\left(\varphi\left(s, x_{0}, u_{0}\right), u_{0}\right) \alpha(s)+ \\
+D_{2} R\left(\varphi\left(s, x_{0}, u_{0}\right), u_{0}\right) v(s) d s
\end{gathered}
$$

analogamente

$$
\beta(t)=\int_{0}^{t} A \beta(s)+D_{2} F(0,0) v(s) d s
$$

e assim,

$$
|\alpha(t)| \leq\|v\|_{\infty}\left(\left\|D_{2} F(0,0)\right\|+M\right)+\int_{0}^{t}(\|A\|+M)|\alpha(s)| d s .
$$

Aplicando a desigualdade de Gronwall em (4.15), obtemos

$$
|\alpha(t)| \leq\|v\|_{\infty}\left(\left\|D_{2} F(0,0)\right\|+M\right) e^{t(\|A\|+M)} .
$$

Usando (4.13) e (4.14) temos que $\forall t \in[0, T]$

$$
\begin{gathered}
|\alpha(t)-\beta(t)|= \\
=\left|\int_{0}^{t} A(\alpha(s)-\beta(s)) d s+\int_{0}^{t} D_{1} R\left(\varphi\left(s, x_{0}, u_{0}\right), u_{0}\right) \alpha(s)+D_{2} R\left(\varphi\left(s, x_{0}, u_{0}\right), u_{0}\right) v(s) d s\right| \leq \\
\leq \int_{0}^{t}\|A\||\alpha(s)-\beta(s)| d s+M\left(\int_{0}^{t}|\alpha(s)| d s+\|v\|_{\infty}\right) .
\end{gathered}
$$

Substituindo (4.16) em (4.17) temos

$$
|\alpha(t)-\beta(t)| \leq
$$




$$
\begin{aligned}
& \leq M\left(\int_{0}^{T}\|v\|_{\infty}\left(\left\|D_{2} F(0,0)\right\|+M\right) e^{T(\|A\|+M)}+\|v\|_{\infty}\right) d s+\int_{0}^{t}\|A\||\alpha(s)-\beta(s)| d s= \\
& =M\|v\|_{\infty}\left(T e^{T(\|A\|+M)}\left(\left\|D_{2} F(0,0)\right\|+M\right)+1\right)+\int_{0}^{t}\|A\||\alpha(s)-\beta(s)| d s= \\
& =C(M)\|v\|_{\infty}+\int_{0}^{t}\|A\||\alpha(s)-\beta(s)| d s
\end{aligned}
$$

onde

$$
C(M):=M\left(T e^{T(\|A\|+M)}\left(\left\|D_{2} F(0,0)\right\|+M\right)+1\right) .
$$

Usando a desigualdade de Gronwall podemos concluir que

$$
|\alpha(T)-\beta(T)| \leq C(M)\|v\|_{\infty} e^{T\|A\|} .
$$

Observe que $\beta(T)$ é a aplicação $\Theta^{\prime}(0)$ definida em (4.7) com $t_{0}$ substituido por $T$ e $B$ substituido por $D_{2} F(0,0)$. Com a hipótese do par $\left(A, D_{2} F(0,0)\right)$ controlável, temos $\beta(t)$ sobrejetora, e como as aplicações sobrejetoras formam um conjunto aberto no espaço das funções lineares contínuas $\mathcal{L}\left(\left(C(0, T), \mathbb{R}^{n}\right), \mathbb{R}^{n}\right)$, existe $r>0$ tal que, qualquer $H \in \mathcal{L}\left(\left(C(0, T), \mathbb{R}^{n}\right), \mathbb{R}^{n}\right)$, com $|H-\beta(T)|<$ $r$, é sobrejetiva.

Como $C(M) \rightarrow 0$ quando $M \rightarrow 0^{+}$, existe $M=M\left(A, D_{2} F(0,0), T\right)$ tal que $|\alpha(T)-\beta(T)| \leq r$, o que implica que $\alpha(t)=D_{3} \varphi\left(T, x_{0}, u_{0}\right)$ é sobrejetiva. Note que esta conclusão independe de $\left(x_{0}, u_{0}\right) \in \mathbb{R}^{n} \times$ intU . Usando o teorema do posto, temos a existência de uma vizinhança $W$ de 0 em $\mathcal{V}_{\left(T, u_{0}\right)}$ tal que $y=\varphi\left(T, x_{0}, u_{0}\right) \in \operatorname{int} \Psi(W) \subset \mathcal{O}_{T}^{+}\left(x_{0}\right)$.

Para mostrar que $\mathcal{O}_{T}^{-}\left(x_{0}\right)$ é aberto, basta observar que $\mathcal{O}_{T}^{-}\left(x_{0}\right)=\tilde{\mathcal{O}}_{T}^{+}\left(x_{0}\right)$, onde $\tilde{\mathcal{O}}_{T}^{+}\left(x_{0}\right)$ é o conjunto de acessibilidade em tempo $T$ do sistema

$$
\dot{x}=-\left(A x+D_{2} F(0,0) u+R(x, u)\right) .
$$

Estamos procurando provar que os conjuntos $\mathcal{O}^{+}(x)$ e $\mathcal{O}^{-}(x)$ são abertos. O lema 4.9 mostra que $\mathcal{O}_{T}^{+}(x)$ e $\mathcal{O}_{T}^{-}(x)$ são abertos, mas note que $M$ depende de $T$. No entanto, o seguinte lema mostra que podemos escolher uma única constante $M$ para todo $T$, o que nos permitirá concluir que os conjuntos $\mathcal{O}^{+}(x)$ e $\mathcal{O}^{-}(x)$ são abertos.

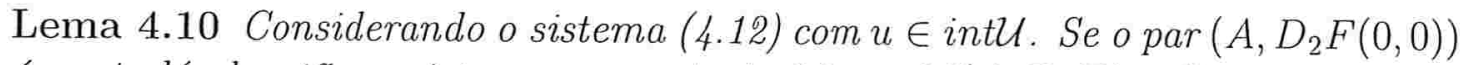
é controlável então, existe uma constante $M:=M\left(A, D_{2} F(0,0)\right)$ tal que, 
para qualquer função $R$ de classe $C^{1}$, com $\left\|D_{1} R\right\|<M$ e $\left\|D_{2} R\right\|<M$ uniformemente, temos: Para qualquer $x_{0} \in \mathbb{R}^{n}$, os conjuntos $\mathcal{O}_{\geq 1}^{+}\left(x_{0}\right)$ e $\mathcal{O}_{\geq 1}^{-}\left(x_{0}\right)$ são abertos.

Demonstração. Dado $x_{0} \in \mathbb{R}^{n}$, escolha $M=M\left(A, D_{2} F(0,0), 1\right)$ dado pela proposição 4.9. Desta forma $\mathcal{O}_{1}^{+}\left(x_{0}\right)$ é um conjunto aberto. Agora, $\forall y \in \mathcal{O}_{\geq 1}^{+}\left(x_{0}\right), y=\varphi(t, x, u)$ para algum $t \geq 1$ e algum $u \in$ intU. Seja $V \subset \operatorname{int} \mathcal{O}_{1}^{+}(x)$ uma vizinhança aberta de $\varphi(1, x, u)$. É claro que $\varphi(t, x, u) \subset$ $\varphi\left(t-1, V, \theta_{1}(u)\right)$, onde $\theta_{1}(u)(t)=u(t+1)$. Como $\varphi\left(t-1, \cdot, \theta_{1}(u)\right): \mathbb{R}^{n} \rightarrow$ $\mathbb{R}^{n}$ é um homeomorfismo, temos que $W:=\varphi\left(t-1, V, \theta_{1}(u)\right)$ é um aberto que contém $y$ e está contido em $\mathcal{O}_{\geq 1}^{+}\left(x_{0}\right)$ o que demonstra que $\mathcal{O}_{\geq 1}^{+}\left(x_{0}\right)$ é um conjunto aberto. Para demonstrar que $\mathcal{O}_{\geq 1}^{-}\left(x_{0}\right)$ é aberto o raciocínio é análogo.

Uma aplicação imediata do lema 4.10 é o seguinte

Teorema 4.11 Considerando o sistema (4.12) com $u \in$ int $\mathcal{U}$, se o par $\left(A, D_{2} F(0,0)\right)$ é controlável então, existe uma constante $M:=M\left(A, D_{2} F(0,0)\right)$ tal que, para qualquer função $C^{1} R$ com $\left\|D_{1} R\right\|<M$ e $\left\|D_{2} R\right\|<M$ uniformemente, temos que $\mathcal{H}_{0}$ é um conjunto aberto não vazio e $\mathcal{H}_{0}$ é o único conjunto de controlabilidade total do sistema (4.12).

Demonstração. Basta observar que $\mathcal{H}_{0}=\mathcal{O}_{\geq 1}^{+}(0) \cap \mathcal{O}_{\geq 1}^{-}(0)$ (proposição 1.6). Do Lema 4.10, resulta então que $\mathcal{H}_{0}$ é aberto. Se usarmos o controle $u \equiv 0$ temos que $0 \in \mathcal{H}_{0}, \operatorname{logo} \mathcal{H}(0) \neq \emptyset$.

Para a unicidade, suponhamos inicialmente que exista outro conjunto de controlabilidade total $\mathcal{H}_{1}$. Da mesma maneira que na demonstração do teorema 4.8, podemos construir uma aplicação contínua $\hat{x}:[0,1] \rightarrow \mathcal{P}$ que associa, a cada $\lambda \in[0,1]$ uma trajetória periódica $x_{\lambda}$ do sistema (4.12) tal que $x_{0}(t) \subset \mathcal{H}_{0}$ e $x_{1}(t) \subset \mathcal{H}_{1}$. Considere $\lambda^{*}=\sup \left\{\lambda \in[0,1]: x_{\lambda} \subset \mathcal{H}_{0}\right\}$.

$\mathrm{O}$ fato de $\mathcal{H}_{0}$ ser aberto, implica que $\lambda^{*}>0$.

Para $\lambda^{*} \in(0,1)$, analizaremos em 2 casos:

$1^{o}$ caso: $x_{\lambda^{*}} \subset \mathcal{H}_{0}$. A escolha do $\lambda^{*}$ implica que $x_{\lambda^{*}+\varepsilon} \nsubseteq \nsubseteq \mathcal{H}_{0}$ para todo $\varepsilon>0$ tal que $\lambda^{*}+\varepsilon<1$. No entando, pelo lema 4.10, $x_{\lambda^{*}} \subset$ int $\mathcal{H}_{0}$ e, dada a continuidade de $\hat{x}, x_{\lambda^{*}+\varepsilon} \subset \mathcal{H}_{0}$ para $\varepsilon$ sufcientemente pequeno, o que é uma contradição.

$2^{\circ}$ caso: $x_{\lambda^{*}} \nsubseteq \mathcal{H}_{0}$. A escolha de $\lambda^{*}$ implica que $x_{\lambda^{*}-\varepsilon} \subset \mathcal{H}_{0}$ para todo $\varepsilon>0$ tal que $\lambda^{*}-\varepsilon>0$. Pelo lema $4.10, x_{\lambda^{*}} \subset$ int $\mathcal{H}_{2}$ para algum conjunto de controlabilidade total $\mathcal{H}_{2}$. Dada a continuidade de $\hat{x}, x_{\lambda^{*}-\varepsilon} \subset \mathcal{H}_{2}$ para $\varepsilon$ sufcientemente pequeno, o que é uma contradição. 
Se $\lambda^{*}=1$ e $x_{1} \nsubseteq \mathcal{H}_{0}$, teremos $x_{1-\varepsilon} \subset \mathcal{H}_{0} \forall \varepsilon \in(0,1]$, que conduz para a mesma contradição do $2^{\circ}$ caso.

Logo, $\lambda^{*}=1$ e $x_{1} \subset \mathcal{H}_{0}$, o que mostra que só existe um conjunto de controlabilidade total.

\subsection{Notas}

Alguns resultados técnicos deste capítulo foram adaptados de [16] e [17], que tratam do problema da unicidade dos conjuntos controláveis. A contribuição desta tese reside em termos considerado os conjuntos de controlabilidade total no lugar dos conjuntos controláveis e estudar separadamente o caso de sistemas não necessariamente localmente acessíveis (teorema 4.8). 


\section{Bibliografia}

[1] Akin, E. : The General Topology of Dynamical Systems - (Graduate Studies in Mathematics ; 1), American Mathematical Society, (1993).

[2] Arnold, L. and Kliemann, W. : Qualitative theory of stochastic systems. Probabilistic Analysis and Related Topics, vol III, Bharucha-Reid (ed.), Academic Press, New York, (1983) 1-79.

[3] Bacciotti, A. and Kalouptsidis, N. : Topological Dynamics of Control Systems: Stability and Attraction, Nonlinear Analysis, Theory, Methods \& Applications, Vol. 10, no. 6, (1986), 547-565.

[4] Banks, J., Brooks, J., Cairns, G. and Stacey, P. : On Devaney's definition of chaos. Am. Math. Month., 99, (1992) 332-334.

[5] Braga Barros, C. J. : Relative invariance for monoid actions. Proyecciones 20 (2001), no. 3, 281-294.

[6] Braga Barros, C. J. : On the number of control sets on flag manifolds of the real simple Lie groups. J. Lie Theory 8 (1998), no. 2, 393-397.

[7] Braga Barros, C. J. and San Martin, L. A. B. On the number of control sets on projective spaces, Sys. Control Lett., vol 29 (1996), 21-26.

[8] Brézis, H : Análisis funcional, Alianza Editorial,1984.

[9] Brockett, R. W. : Systems theory on group manifolds and coset spaces. SIAM J. Control Opt., vol 10 (1972) 265-284.

[10] Colonius, F. and Kliemann, W. : Some Aspects of Control Systems as Dynamical Systems. Journal of Dynamics and Differential Equations, vol. 5, 3 (1993) 469-494. 
[11] Colonius, F. and Kliemann, W. : Limit Behavior and Genericity for Nonlinear Control Systems. Journal of Differential Equations, vol. 109, (1994) 8-41.

[12] Colonius, F. and Kliemann, W. : Controllability and stabilization of onedimensional systems near bifurcation points. Systems \& Control Letters, vol. 24, (1995) 87-95.

[13] Colonius, F. and Kliemann, W. : The Lyapunov spectrum of families of time varying matrices, Trans. Amer. Math. Soc., vol 348 (1996) 43894408.

[14] Colonius, F and Kliemann, W. : The Morse spectrum of linear flow on vector bundles, Trans. Amer. Math. Soc., vol 348 (1996) 4355-4388.

[15] Colonius, F. and Kliemann, W. : The Dynamics of Control, Birkhäuser, 2000.

[16] Colonius, F. and Spadini, M. Local Uniqueness of control sets for nonlinear systems, Stability and Stabilization of Nonlinear Systems, D. Aeyels, F. Lamnabhi-Lagarrigue, and A. van der Schaft, eds., SpringerVerlag, (1999) 115-135.

[17] Colonius, F. and Spadini, M. Local Control Sets. Pré-print.

[18] Devaney, R. L. : An Introduction to Chaotic Dynamical Systems, Benjamin/Cummings, Menlo Park, CA, 1986.

[19] Dunford \& Schwartz : Linear Operators - Part I: General Theory, John Wiley \& Sons, 1988.

[20] Engelking, R : General Topology - (Sigma series in Pure Mathematics ; 6), Heldernann Verlag Berlin, 1989.

[21] Gayek, J.E. and Vincent, T.L. : On the intersection of controllable and reachable sets, Journal of Optimization Theory and Applications, Vol 50, no. 2, (1986), 267-278.

[22] Hermes, H. : On local and global controllability, SIAM J. Control Opt., vol 12 (1974) 252-261. 
[23] Isidori, Alberto : Nonlinear Control Systems $3^{a}$ ed. - (Communications \& Control Engineering Series), Springer-Verlag, 1995.

[24] Jurdjevic, V. and Sussmann, H. : Control Systems on Lie Groups, Journal of Differential Equations, vol. 12, (1972) 313-329.

[25] Jurdjevic, V : Geometric Control Theory - (Cambridge Studies in Advanced Mathematics ; 52), Cambridge University Press, 1997.

[26] Kang, W : Bifurcation and normal form of nonlinear control systems I SIAM J. Control Optim., 36, no 1, (1998) 193-212.

[27] Kelley, J. L. : General Topology - (Graduate Text in Mathematics 27), Springer-Verlag (1985).

[28] Lee, E. B. and Marcus, L. : Fundations of Optimal Control Theory, John Wiley \& Sons, 1967.

[29] Lobry, C. : Contrôlabilité des systèmes non linéaires, SIAM J. Control Opt., vol 8 (1970) 573-605.

[30] Mañe, R. : Introdução à Teoria Ergódica - (Projeto Euclides), IMPA, 1983.

[31] Panasiuk, A.I. : Dynamics of Sets Defined by Differential Inclusions, Siberian Math. J., vol. 25, no. 5, (1986), 757-765.

[32] Roxin, E.O. : Stability in general control systems, Journal of Differential Equations 1, (1965), 115-150.

[33] Roxin, E.O. : Control Theory and its Applications - (Stability and Control: Theory, Methods and Applications ; 4), Gordon and Breach Science Publishers, 1997.

[34] Roxin, E.O. : Reachable sets, limit sets and holding sets in control systems, Nonlinear Analysis and Applications, V, V. Lakshmikantham ed., Marcel Dekker Inc. Publ., New York, 1987, 533-540.

[35] Roxin, E.O. : Application of Holding Sets to Optimal Control, Proceedings of The International Conference on Theory and Applications of Differential Equations, Ohio University (1988), 346-349. 
[36] Roxin, E.O. : The Attainable Set in control systems, Lect. Notes in Pure and Appl. Math.,142, (1993), 307-319.

[37] Roxin, E.O. : The Attainability Order in control systems, Lect. Notes in Pure and Appl. Math.,152, (1994), 793-798.

[38] San Martin, L. A. B. : Invariant Control Sets on Fibre Bundle. PhD thesis, Warwick University (1986).

[39] San Martin, L. A. B. and Tonelli, P. A. Semigroup Actions on homogeneous spaces. Semigroup Forum, vol 14 (1994) 1-30.

[40] San Martin, L. A. B. : Órbitas de familias de campos de vetores, (1999), http://www.ime.unicamp.br/ ${ }^{\sim}$ smartin/

[41] San Martin, L. A. B. : Maximal semigroups in semi-simple Lie groups. Tans. Am. Math. Soc., 353 (2001), no. 12, 5165-5184.

[42] Sell, G.R. : Topological Dynamics and Ordinary Differential Equations, Van Nostrand Reinhold Company, London, 1971.

[43] Sussmann, H. J. Orbits of families of vectors fields and integrability of distribuitions. Trans. Am. Math. Soc., vol 180 (1973) 171-188.

[44] Tonelli P.A. : Control Sets on Homogeneous Spaces, Dissertation, University of Bremen, (1991).

[45] Vincent T.L. : Renewable Resource Management - (Lecture Notes in Biomathematics ; 40), Springer-Verlag, 1981.

[46] Wirth, F. : Robust stability of discrete-time systems under time-varying perturbations, Dissertation, University of Bremen, (1995). 